San Jose State University

SJSU ScholarWorks

Master's Theses

Master's Theses and Graduate Research

Summer 2019

\title{
Time-Invariant Tensile Necking of Blood on an Electrowetting Digital Microfluidic Platform
}

Curtis Young

San Jose State University

Follow this and additional works at: https://scholarworks.sjsu.edu/etd_theses

\section{Recommended Citation}

Young, Curtis, "Time-Invariant Tensile Necking of Blood on an Electrowetting Digital Microfluidic Platform" (2019). Master's Theses. 5053.

DOI: https://doi.org/10.31979/etd.rpfx-4uq3

https://scholarworks.sjsu.edu/etd_theses/5053

This Thesis is brought to you for free and open access by the Master's Theses and Graduate Research at SJSU ScholarWorks. It has been accepted for inclusion in Master's Theses by an authorized administrator of SJSU ScholarWorks. For more information, please contact scholarworks@sjsu.edu. 
TIME-INVARIANT TENSILE NECKING OF BLOOD ON AN ELECTROWETTING DIGITAL MICROFLUIDIC PLATFORM

\author{
A Thesis \\ Presented to \\ The Faculty of the Department of Mechanical Engineering \\ San José State University \\ In Partial Fulfillment \\ of the Requirements for the Degree \\ Master of Science \\ by \\ Curtis J. Young
}

August 2019 
(C) 2019

Curtis J. Young

ALL RIGHTS RESERVED 
The Designated Thesis Committee Approves the Thesis Titled

TIME-INVARIANT TENSILE NECKING OF BLOOD ON AN ELECTROWETTING DIGITAL MICROFLUIDIC PLATFORM

by

Curtis J. Young

APPROVED FOR THE DEPARTMENT OF MECHANICAL ENGINEERING

SAN JOSÉ STATE UNIVERSITY

August 2019

Sang-Joon Lee, Ph.D. Department of Mechanical Engineering

Anand Ramasubramanian, Ph.D. Department of Chemical and Materials Engineering

Melinda Simon, Ph.D.

Department of Biomedical Engineering 


\section{ABSTRACT \\ TIME-INVARIANT TENSILE NECKING OF BLOOD ON AN ELECTROWETTING DIGITAL MICROFLUIDIC PLATFORM \\ by Curtis Young}

Recent developments in electrowetting-on-dielectric (EWOD) technology have expanded the possibilities for testing methods and investigation of blood. This work evaluated the development of necking geometry of whole blood and red blood cell (RBC) suspensions on a digital microfluidic (DMF) platform. This was achieved by applying tension to liquid droplet specimens on an EWOD-based device using applied electrowetting forces, thereby inducing necking. The deformation of $5 \mu \mathrm{L}$ fluid specimens was recorded using neck radius and minimum neck width as two characteristic geometric parameters. Tensile tests were performed on phosphate-buffered saline (PBS), whole blood diluted in PBS, and RBCs suspended in PBS at hematocrit values of 10\%. Although rheological measurements suggested higher stiffness of RBC suspensions when treated with neuraminidase, necking profiles were indistinguishable from those of untreated RBC suspensions. When tested at two different dilutions, whole blood diluted 1:20 and 1:10 in PBS exhibited distinct necking profiles. The necking development was observed to be time-invariant and insensitive to the large variability otherwise observed among distinct DMF chips and distinct locations on the chips. 


\section{ACKNOWLEDGMENTS}

This work was supported in part by an AHA Institutional Research Enhancement Award (18AIREA33960524) from the American Heart Association. Many thanks to Dr. Sang-Joon Lee, thesis advisor and committee chair and committee members Dr. Anand

Ramasubramanian and Dr. Melinda Simon. We would also like to express appreciation to Dr. Ryan Fobel of Sci-Bots, Inc., for application-specific advice related to electrowetting of blood on a DMF platform, Erika Young for assistance in the operation of the DropBot platform, Suyog Pathare and Chaitanya Puglianda for assistance acquiring and processing blood specimens, Sridhar Nyshadham for assistance performing rheometry experiments, and Jason Wong for assistance in image feature extraction. 


\section{TABLE OF CONTENTS}

List of Tables............................................................................ vii

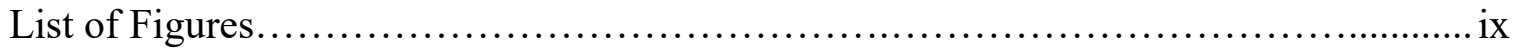

List of Symbols and Abbreviations................................................. xi

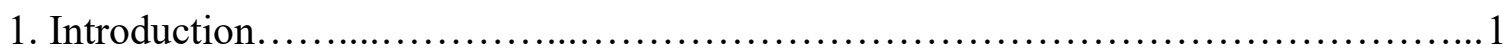

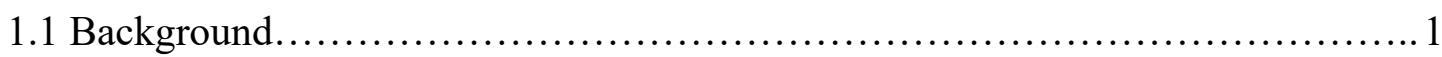

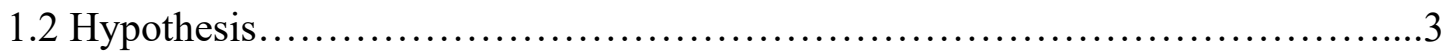

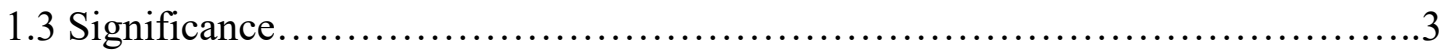

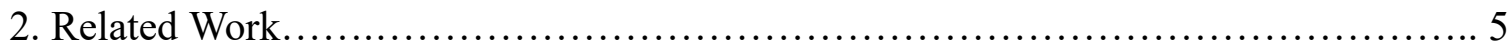

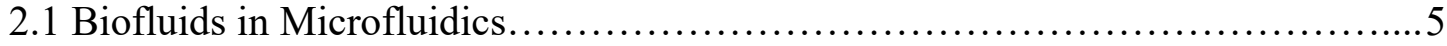

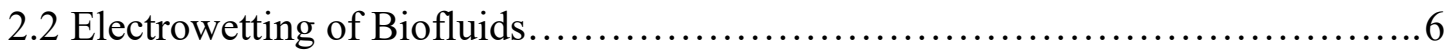

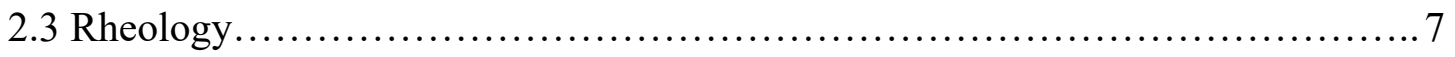

2.3.1 Microfluidic Rheometry .........................................

2.3.2 Neuraminidase Treatment for RBC Surface Charge Removal................ 8

2.3.3 Extensional Flow Rheometry......................................... 9

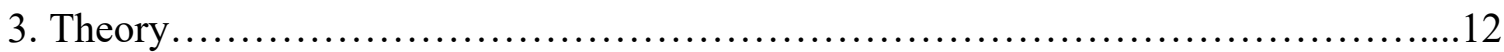

3.1 Electrowetting Forces.................................................. 12

3.2 Dielectrophoretic Forces..................................................13

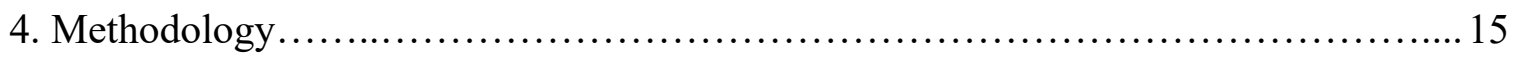

4.1 Experimental Assembly.................................................. 15

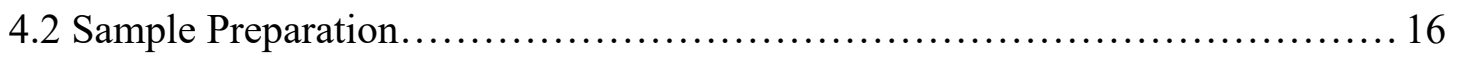

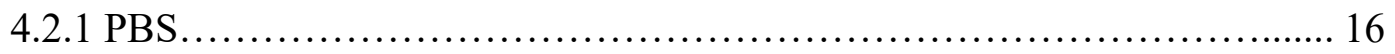

4.2.2 Whole Blood..................................................... 17

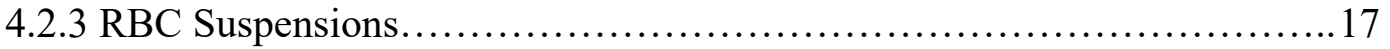

4.2.4 Neuraminidase Treatment.......................................... 18

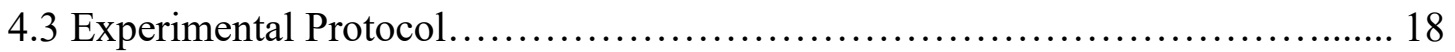

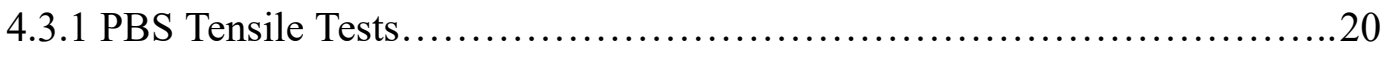

4.3.2 RBC and Whole Blood Tensile Tests....................................21 


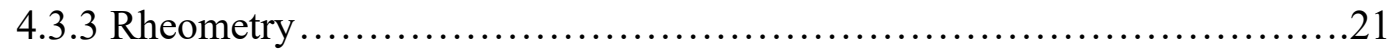

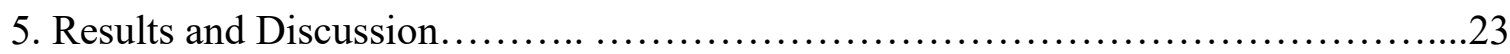

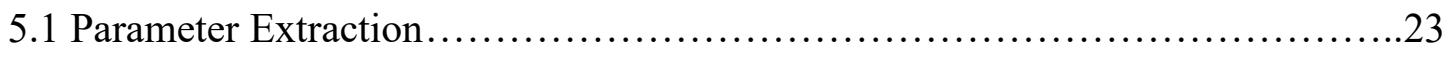

5.2 Time Development of the Necking Profile .....................................25

5.3 Time-Invariant Development of Necking Profile ..............................27

5.4 Neuraminidase Treatment of Red Blood Cells ...................................32

5.5 Estimation of Electrowetting Force............................................ 34

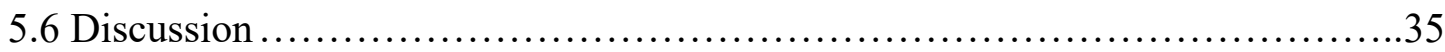

5.6.1 Variability in Tensile Behavior......................................... 35

5.6 .2 Component Separation................................................

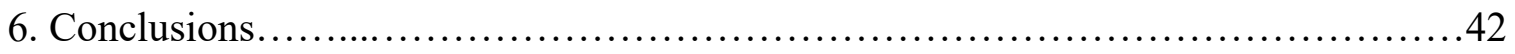

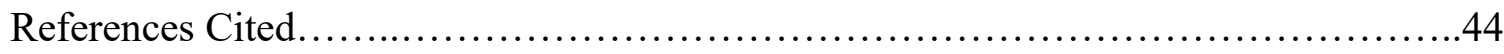

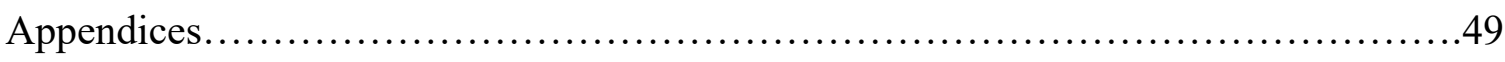

Appendix A: Experimental Table...............................................49

Appendix B: Electronic Lab Notebook..........................................50 


\section{LIST OF TABLES}

Table 1. Parameter Values for Exponential Fits to Tensile Tests.........................31 


\section{LIST OF FIGURES}

Figure 1. DMF chip electrode layout describing the locations of the test sites....... 15

Figure 2. Electrode sequence to actuate liquid into position for tensile test on-chip.. 19

Figure 3. Electrode sequence to perform apply tension to liquid on -chip............ 20

Figure 4. Example of time progression of necking during tensile test............. 23

Figure 5. Illustration of necking parameters.................................. 24

Figure 6. Neck width versus time for PBS $\quad$................................ 25

Figure 7. Neck width versus time for whole blood diluted $1: 20 \ldots \ldots \ldots \ldots \ldots \ldots \ldots .26$

Figure 8. Neck width versus time for untreated RBCs........................ 27

Figure 9. Neck radius versus neck width for PBS.......................... 28

Figure 10. Neck radius versus neck width for whole blood diluted 1:20........... 29

Figure 11. Neck radius versus neck width for whole blood diluted $1: 10 \ldots \ldots \ldots \ldots . .29$

Figure 12. Neck radius versus neck width for untreated RBCs................... 30

Figure 13. Neck width versus neck radius for neuraminidase-treated RBCs.............31

Figure 14. Microscope images comparing untreated and neuraminidase treated red blood cells in plasma............................................. 32

Figure 15. Microscope images comparing untreated and neuraminidase treated red blood cells suspended in plasma and saline.......................... 33

Figure 16. Shear stress versus shear strain for RBC suspensions.................. 34

Figure 17. Separation during tensile testing on whole blood diluted $1: 10 \ldots \ldots \ldots \ldots \ldots . . .38$ 
Figure 18. Separation of untreated RBC suspension over two consecutive tensile

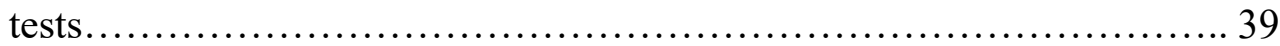

Figure 19. Separation of neuraminidase-treated RBC suspension over two consecutive tensile tests........................................... 41 


\section{LIST OF SYMBOLS AND ABBREVIATIONS}

$\begin{array}{lll}\text { Symbol } & \text { Units } & \text { Description } \\ C & \mathrm{~F} / \mathrm{m}^{2} & \text { capacitance } \\ V & \mathrm{~V} & \text { voltage } \\ \gamma & \mathrm{N} / \mathrm{m} & \text { surface tension } \\ \theta & \circ & \text { contact angle } \\ \theta_{0} & \circ & \text { non-voltage contact angle } \\ \varepsilon & \mathrm{F} / \mathrm{m} & \text { permittivity } \\ \varepsilon_{0} & \mathrm{~F} / \mathrm{m} & \text { permittivity of free space } \\ f_{w} & \mathrm{~N} / \mathrm{m} & \text { capillary line force density } \\ h & \mathrm{~m} & \text { layer height } \\ F & \mathrm{~N} & \text { force } \\ E & \mathrm{~W} & \text { energy } \\ L_{e l} & \mathrm{~m} & \text { electrode length } \\ f & \mathrm{~Hz} & \text { frequency } \\ x & \mathrm{~m} & \text { general spatial dimension } \\ f_{C} & \mathrm{~Hz} & \text { critical frequency } \\ g_{L} & \mathrm{~S} & \text { liquid conductance } \\ T & { }^{\circ} \mathrm{C} & \text { Temperature } \\ p & \mathrm{~Pa} & \text { pressure } \\ H & \% & \text { Hematocrit } \\ R & \mathrm{~m} & \text { neck radius } \\ d & \mathrm{~m} & \text { minimum neck width } \\ & & \end{array}$

$\begin{array}{ll}\text { Abbreviation } & \text { Full Spelling } \\ \text { EWOD } & \text { electrowetting-on-dielectric } \\ \text { DMF } & \text { digital microfluidic } \\ \text { PBS } & \text { phosphate-buffered saline } \\ \text { RBC } & \text { red blood cell } \\ \text { WBC } & \text { white blood cell } \\ \text { ITO } & \text { indium-tin oxide } \\ \text { FFP } & \text { fresh frozen plasma } \\ \text { RCF } & \text { relative centrifugal force }\end{array}$




\section{INTRODUCTION}

\subsection{Background}

Blood plays many vital roles within the human body. It comprises several major components: red blood cells (RBCs) transport oxygen and carbon dioxide between the lungs and tissue, white blood cells (WBCs) fight infection and regulate immune function, platelets help form clots, and plasma contains clotting proteins and nutrients [1], [2]. Electrowetting-based digital microfluidic (DMF) devices provide a platform for novel methods of investigating blood which can offer advantages over current analysis techniques. Electrowetting-based DMF platforms have seen significant recent development especially as "lab-on-a-chip" devices that create, combine, move, and split droplets of liquid [3]. Such platforms operate based on the principles of electrowetting, which involves the modification of the contact of liquid on a surface using an applied electric field [4]. Electrowetting was first harnessed when Lippmann used externally-added electrostatic charge to alter capillary forces at the interface of polarized mercury-electrolyte to construct an electrometer [5], and modern applications of electrowetting include adjustable liquid lenses, electronics displays, and droplet manipulation platforms [3], [6], [7].

The contact of a fluid on a surface can be described by the wetting angle, or the contact angle between the surface and the profile of the fluid droplet. A fluid-surface contact with a wetting angle greater than $90^{\circ}$ is described as hydrophobic and a contact with a wetting angle less than $90^{\circ}$ is described as hydrophilic [8]. Electrowetting-on 
dielectric (EWOD) devices utilize an applied voltage through a conductive surface with an additional layer acting as a dielectric between the fluid and conductive layer [9]. When an electrowetting voltage is applied over the droplet to the conductive layer, a parallel plate capacitor is formed at the interface, ultimately reducing the wetting angle of the fluid. As such, an electrowetting voltage reduces the hydrophobicity of a fluid-surface contact.

Fluid droplet manipulation can be achieved by harnessing this behavior using a surface with multiple independent, adjacent electrodes. When a droplet is positioned over an electrode, applying a voltage over an adjacent electrode reduces the hydrophobicity of the fluid locally over the actuated electrode. If the resulting driving force within the droplet overcomes the shear and viscous drag forces, the droplet will move onto the actuated electrode. Using this principle of droplet actuation, droplet operations such as mobilization, splitting, and mixing are made possible. Droplet actuation on an open surface can prove to be demanding, requiring excessively high voltages and performing inconsistently. Accordingly, many modern EWOD droplet manipulation platforms use designs which reduce the actuation requirements [10]. A hydrophobic layer is often applied over the insulating dielectric layer, reducing the drag on the droplet. In addition, two-plate designs are commonly used, employing a top plate with a conductive layer and hydrophobic coating.

Key advantages that EWOD platforms offer over mechanical testing systems for biofluids are reduced reagent volume and improved mixing consistency. Actuation with 
electrical signals over patterned electrodes on the platform can create and manipulate precise, microliter-scale droplets and mix reagents into far less heterogeneous combinations than conventional methods [11]. In addition, EWOD platforms offer simplicity relative to other microfluidic systems, as they use only electrical signals on a patterned surface rather than pumps, valves, or other mechanical components. This allows for lower-cost fabrication, greater portability, and more convenient automation of operations [3], [12], [13].

\subsection{Hypothesis}

The hypothesis of this work is that blood and RBC suspensions will have a characteristic curve based on necking geometry which is robust against variability in the temporal development during on-chip testing. The rationale for this hypothesis is that the temporal response of liquids to electrowetting forces can be highly dependent on device-specific variability in features such as gap spacing and hydrophobic coating uniformity, yet ultimately a liquid will still tend toward a lowest-energy equilibrium shape.

\subsection{Significance}

An increased understanding of blood and its components leads to the development of treatments for diseases. For instance, anemia is a dangerous condition characterized by a lack of functioning red blood cells. Anemia is correlated with severe complications in cardiovascular disease such as stroke and venous thrombosis, but the mechanisms behind this effect are not well understood [1], [14], [15]. A method of investigation on a digital 
microfluidic platform presents options for point-of-care testing for anemia. This would expand the capabilities of current assays as well as allow new assays to be devised based on the digital microfluidic platform. While the use of biofluids on a digital microfluidic platform has been investigated, a time-invariant method of investigating the geometry of liquid specimens has not been explored. This can mitigate issues of variability between addressable sites on a digital microfluidic device. While the role of RBC surface charge is well-studied with regards to its effect on agglutinability [16] and its consequent influence on blood rheometry, investigation on a DMF device reveals the behavior of charged and uncharged cells during flow within an electric field. The use of a DMF platform means RBC suspensions can be compared with high spatial and temporal resolution, revealing previously undiscovered differences in their behavior. 


\section{RELATED WORK}

\subsection{Biofluids in Microfluidics}

To combine reagents on a digital microfluidic platform, droplet mixing operations are necessary. Paik et al. determined that in linear array mixing, aspect ratio of droplet specimens plays an important role [17]. In aspect ratios ranging from 0.2 to 0.66 , an aspect ratio of 0.4 was found to produce optimal mixing results, and 0.2 the worst mixing results. Droplet samples ranged from $2350 \mathrm{~nL}$ to $800 \mathrm{~nL}$, with gap heights ranging from $600 \mu \mathrm{m}$ to $1 \mathrm{~mm}$, and mixing times ranging from $1.7 \mathrm{~s}$ to $14.9 \mathrm{~s}$. Flow reversibility effects have been shown to hinder mixing, and methods to reduce this were explored using larger electrode arrays with the aspect ratio of 0.4 . Using a $2 \times 4$ electrode array with a droplet-pivot mixing method resulted in a mixing time of $2.9 \mathrm{~s}$ for two $1.4 \mu \mathrm{L}$ droplets. The EWOD device used actuated droplets in silicone oil at $50 \mathrm{~V}$ with a frequency of 16 Hz. Mixing results were obtained by combining a fluorescent droplet with a stationary, non-fluorescent droplet and subsequent image processing.

While radial droplet stressing through electrowetting has been regular practice, longitudinal droplet stressing has been uncommon. Much of the established literature concerning this mode of droplet stressing and deformation has been related to droplet cutting. Cho et al. achieved droplet cutting by deforming a droplet longitudinally over a line of three electrodes [10]. In this configuration, the two outer electrodes were activated while the center electrode is grounded. This caused the contact angle of the droplet at 
each end to decrease as the local radii of curvature increase. As the droplet volume must stay constant, the meniscus over the center electrode pinched, initiating the cut.

\subsection{Electrowetting of Biofluids}

Barbulovic-Nad et al. demonstrated the use of an electrowetting-based device for biofluids, specifically in implementing cell-based assays [18]. Each EWOD platform used chromium/gold electrodes, a Parylene-C dielectric layer of $2 \mu \mathrm{m}$, and a Teflon-AF hydrophobic layer of $50 \mathrm{~nm}$. Using AC voltages of up to $140 \mathrm{~V}$ and a two-plate assembly, Jurkat T-cells were combined with a reagent in $150 \mathrm{~nL}$ droplets. There were no observed effects on cell biochemistry due to the actuation, suggesting DMF devices have potential in similar applications.

Li et al. explored electrowetting on a blood droplet using an open configuration device and wire electrode with rabbit's blood [19]. The blood droplet's change in contact angle was measured in response to the applied electrowetting force. Over an applied voltage range from $0 \mathrm{~V}$ to $60 \mathrm{~V}$, the blood droplet changed wetting angle from $106^{\circ}$ to 85.9 $9^{\circ}$. Additionally, an EWOD platform was used by Mais et al. to extract analytes in order to quantify amino acids in dried blood spot samples, actuating extraction solvent and extracted amino acid solution on a two-plate DMF device [20] and $\mathrm{Ng}$ et al. actuated dilute whole blood on a digital microfluidic platform for remote serological immunoassays [21]. 


\subsection{Rheology}

Blood is a non-Newtonian and viscoelastic substance. The rheology of blood is influenced by plasma viscosity as well as RBC behavior [22], [23]. Under low-flow conditions, RBCs form aggregates, which are highly influential to blood flow in low-shear regions. Initially, linear stacks of cells formed, a phenomenon known as rouleaux formation. The rouleaux stacks then interact to form larger, three-dimensional structures [24]. The size of these aggregates is inversely proportional to the shear forces, as they are dispersed with increasing shear forces. The cell deformability and orientation of RBCs are the primary cellular factors influencing blood fluidity at high shear rates [25]-[27]. Due to the contribution of RBC behavior to blood rheology, blood viscosity increases exponentially with hematocrit [28].

\subsubsection{Microfluidic Rheometry}

Microfluidics present opportunities to mitigate issues inherent to macroscale shear rheometry, including low-viscosity fluids, interfacial artifact effects, and small sample volumes [29]. Microfluidic rheometry devices can measure shear viscosity or extensional properties of a fluid. Examples of microfluidic shear rheometry include methods based on pressure sensing, flow rate sensing, surface tension, and diffusion in a microchannel. The extensional properties of a fluid can be investigated using methods such as stagnation point flow or contraction geometries [29], [30]. Each method has a range of effective operation for both shear rate and viscosity. General challenges faced by microfluidic rheometry methods include cleanability and biofouling fluids, increasing an inherently 
low throughput, integrating optical devices, and operating with a broad range of shear rates [31].

Banpurkar et al. explored the use of electrowetting as a method for performing rheometry experiments on a microfluidic chip [32]. This was done by measuring the electrowetting response of gelatin solutions. An open electrowetting platform was fabricated by patterning an indium tin oxide (ITO)-coated glass slide and coating with Teflon AF 1600. Aqueous droplets were dispensed on the electrowetting platform, and a platinum wire was inserted into the droplet to form a circuit. AC current ranging from 0 to $110 \mathrm{~V}_{\mathrm{rms}}$ was applied to the droplets at $10 \mathrm{kHz}$. As the droplets entered the gel state, the change in contact angle became less pronounced. A model taking into account interfacial energies, electrostatic energy, and deformation energy was developed and fitted to experimental data, ultimately obtaining the elastic modulus. The results were compared to "macro" oscillatory rheometry, specifically the measured storage modulus. The electrowetting rheometry elastic modulus matched well with the macro rheometry storage modulus, but it was noted that the strain field was significantly less uniform in the electrowetting experiment.

\subsubsection{Neuraminidase Treatment for RBC Surface Charge Removal}

Neuraminidase is an enzyme that removes sialic acid on the surface of RBCs, reducing negative surface charge and increasing the cells' tendency to aggregate. This affects the rheology of RBCs [16]. Jan and Chien studied the role of RBC surface charge in electrophoretic mobility and cell agglutinability, specifically the effects of different 
dextrans on neuraminidase-treated RBCs [33]. When suspended in saline, RBCs were not observed to have any increased aggregation. However, when various dextrans (Dextran 20, Dextran 40, Dextran 80) were added, a dramatic difference in aggregation was observed between treated and untreated cells. Wen et al. further examined the influence of neuraminidase treatment on the rheology of RBCs using rabbit cells suspended in plasma at $\mathrm{H}=35 \%$ [34]. Cell aggregate sizes were measured for a range of neuraminidase doses $(0 \mathrm{mU} / \mathrm{mL}$ up to $100 \mathrm{mU} / \mathrm{mL}$ ) as well as a range of incubation times (30 min to 90 $\min$ ) at a dosage of $75 \mathrm{mU} / \mathrm{mL}$. The viscosity was also determined for RBCs over the previous range of neuraminidase dosages using cone-plate rheometry. The viscosity at both low shear rate $\left(10 \mathrm{~s}^{-1}\right)$ and high shear rate $\left(150 \mathrm{~s}^{-1}\right)$ was shown to increase with neuraminidase dosage.

\subsubsection{Extensional Flow Rheometry}

The use of an applied tensile force on a viscoelastic fluid has been explored for use as a rheometry method. Extensional rheometry can dramatically simplify experimental methods relative to shear rheometry. However, any filament-stretching extensional method will cause a non-constant stress in the sample in response to a constant applied force due to decreasing cross-sectional area. Matta and Tytus investigated the rapid stretching of a liquid filament under constant tensile force [35]. This was done by inserting a liquid specimen between the ends of two cylinders oriented vertically. The lower cylinder was dropped, stretching the liquid. The elongational viscosity was determined for viscous Newtonian liquids and viscoelastic liquids by imaging the liquid 
stretching and calculating the stress and deformation rate. This liquid stretching method proved suitable for measuring the extensional viscosity of Newtonian liquids. For viscoelastic liquids, the ratio of stress and deformation rate increased at a rate dependent on the rate of applied stress.

Szabo et al. later subsequently devised a method using this falling cylinder technique to characterize the extensibility of a polymer solution rather than directly determine the extensional viscosity [36]. In this work, the viscoelastic properties of the polymer solution were explored by applying large strains and strain rates to the liquid. Two kinematic descriptions, one using an axially-uniform filament approximation and another using a quasi-two-dimensional Lagrangian model of the elongating filament, were used with a numerical model to obtain an estimate of the maximum extensibility of the polymeric chains in the solution. Additionally, an expression was developed to extract the extensibility estimate from geometry parameters of the filament, which could easily be measured visually. Specifically, an extensibility parameter was found to depend directly on the ratio of the nominal liquid radius and a specific filament radius.

Sousa et al. investigated the behavior of human blood in uniaxial extensional flow [37]. In this work, the filament thinning of blood specimens was observed by inducing capillary breakup of blood specimens. Similar to the cylinder method, the capillary breakup was observed by placing whole blood between the ends of two rods, which were then moved apart at a constant velocity of $10 \mu \mathrm{m} / \mathrm{s}$. The time evolution of the minimum filament diameter for whole blood was measured using high-speed imaging techniques. 
From this, the blood relaxation time was calculated using the exponential decay of the filament diameter in the elasto-capillary regime [38]. For hematocrit levels ranging from $38.7 \%$ and $46.3 \%$, it was shown the variation of extensional relaxation time was minimal. The average extensional relaxation time in air was $114 \mu \mathrm{s} \pm 30 \mu \mathrm{s}$ and $259 \mu \mathrm{s} \pm 47 \mu \mathrm{s}$ in silicone oil. Additionally, increasing the RBC concentration was shown to increase the bulk viscosity, which slowed filament thinning and decreased the necking rate of the specimen. In RBCs suspended in phosphate-buffered saline (PBS), no significant cell aggregation was observed, but RBCs suspended in plasma revealed a small level of aggregation. Particularly near the breakup the filament, a highly heterogeneous distribution of cells was observed in tested specimens, with some distinct aggregates. 


\section{THEORY}

\subsection{Electrowetting Forces}

The driving forces on the droplet can be estimated using the Young-Lippmann equation, which relates contact angle and applied voltage on a droplet [39], [40]. The Young-Lippmann equation can be obtained in a form which describes the relationship between the static contact angle $\theta$, the non-voltage contact angle $\theta_{0}$, each layer's permittivity $\varepsilon_{1}$ and $\varepsilon_{2}$, each layer's thickness $d_{1}$ and $d_{2}$, the applied voltage $V$, the surface tension of the medium $\gamma$, and the permittivity of a vacuum $\varepsilon_{0}$ [5], [41], [42].

$$
\cos \theta=\cos \theta_{0}+\frac{C}{2 \gamma} V^{2}
$$

In the case of dielectric and hydrophobic layers as part of the EWOD platform, the capacitance per unit area can be calculated in terms of each layer's relative permittivity $\varepsilon_{i}$ and each layer's thickness $h_{i}$, and the permittivity of a vacuum $\varepsilon_{0}$.

$$
C=\frac{\varepsilon_{0}}{\sum_{i} h_{i} / \varepsilon_{i}}
$$

In this model, the droplet movement is driven by capillary force on the triple line of contact line between the droplet, substrate, and medium. The capillary line force density $f_{w}$ is given by the contact angle $\theta$ and surface tension $\gamma$ [42].

$$
f_{w}=\gamma\left(\cos \theta-\cos \theta_{0}\right)
$$

This can be integrated over the contour line $L$ to obtain the driving capillary force $F$ on the droplet in a direction of unit vector $\vec{i}_{x}$, where $\vec{n}$ is the unit normal vector to the contact line, and $d l$ is the unit element of the contour line. 


$$
F=\int_{L} \gamma\left(\cos \theta-\cos \theta_{0}\right) d l \vec{n} \cdot \vec{i}_{x}
$$

Integrating over the contact line results in the capillary driving force on the droplet.

$$
F=L \frac{\varepsilon_{0} \varepsilon_{1} \varepsilon_{2} V^{2}}{2\left(\varepsilon_{1} h_{2}+\varepsilon_{2} h_{1}\right)}
$$

This approach has several shortcomings. In DMF applications, driving forces are largely due to electrostatic forces rather than a change in the contact angle. Furthermore, the thermodynamic approach does not consider liquid dielectrophoretic forces [39].

\subsection{Dielectrophoretic Forces}

Liquid dielectrophoretic forces are caused by an electric field gradient within the droplet in a two-plate DMF chip. This occurs due to the voltage difference from the electrode to the top plate, with the droplet between the two [39], [43]. Using an electromechanical model of the DMF platform, the capacitively stored energy in the system can be determined as a function of position and frequency. Equation (6) gives the stored energy for liquid being actuated within a filler substance, where $E$ is the stored energy, $f$ is the frequency of the electrical signal, $x$ is a spatial dimension on plane of the

chip, $L_{e l}$ is the electrode length, $V_{\text {liquid }}$ and $V_{\text {filler }}$ are the voltage values for liquid and filler respectively, and $\varepsilon_{\text {liquid }}$ and $\varepsilon_{\text {filler }}$ are the permittivity values for the liquid and filler, respectively [44].

$$
E(f, x)=\frac{L_{e l}}{2}\left[x \sum_{i} \frac{\varepsilon_{0} \varepsilon_{\text {liquid }} V_{\text {liquid }}^{2}(j 2 \pi f)}{h_{i}}+\left(L_{e l}-x\right) \sum_{i} \frac{\varepsilon_{0} \varepsilon_{\text {filler }} V_{\text {filler }}^{2}(j 2 \pi f}{h_{i}}\right.
$$


The force on the droplet is the derivative of the energy with respect to the droplet position $x$, resulting in the droplet force as a function of the signal frequency and voltage [39].

$$
\begin{gathered}
F(f)=\frac{\partial E(f, x)}{\partial x} \\
F(f)=\frac{L}{2}\left[\sum_{i} \frac{\varepsilon_{0} \varepsilon_{\text {liquid }} V_{\text {liquid }}^{2}(j 2 \pi f)}{h_{i}}-\sum_{i} \frac{\varepsilon_{0} \varepsilon_{\text {filler }} V_{\text {filler }}^{2}(j 2 \pi f)}{h_{i}}\right]
\end{gathered}
$$

A critical frequency $f_{c}$ can be determined based on the device's geometry and materials. When operating at or under this critical frequency, the dominant cause of driving forces on the droplet is accumulated charge at the triple line. When above $f_{c}$, the dominant cause of driving forces on the droplet is the electric field gradient inducing dielectrophoretic forces pulling the droplet towards the electrode [39]. Because of this, the thermodynamic electrowetting model applies while below $f_{c}$, and the dielectrophoretic model applies while above $f_{c}$. The critical frequency $f_{c}$ is given as a function of the capacitance and conductance of elements of the system where $g_{L}$ is the liquid conductance, $C_{p t}$ is capacitance of the lower plate hydrophobic and dielectric layer, $C_{L}$ is the liquid capacitance, and $C_{t}$ is the capacitance of the top layer hydrophobic [44].

$$
f_{c}=\frac{1}{2 \pi} \frac{g_{L}\left(C_{p t}+C_{t}\right)}{\left(C_{p t} C_{L}+C_{t} C_{L}+C_{p t} C_{t}\right)}
$$




\section{METHODOLOGY}

Tensile tests were performed on an EWOD-based DMF platform (DB3-120, Sci-Bots Inc., Kitchener, Ontario, Canada). Experiments were performed on PBS, whole blood diluted in PBS, and RBCs suspended in PBS at $\mathrm{H}=10 \%$. All experiments were performed on DMF chips (Sci-Bots).

\subsection{Experimental Assembly}

The DropBot DB3-120 system [45] was used as the primary DMF platform for this experiment. The system includes a DropBot DB3-120 instrument, a $12 \mathrm{~V}$ power supply, a test board, USB A/B cable, three 90-pin DMF chips, and a device spacer. The DropBot instrument used an integrated high-voltage source for a maximum of $140 \mathrm{~V}_{\mathrm{rms}}$ at $100 \mathrm{~Hz}$ to $10 \mathrm{kHz}$. The system was controlled by open source MicroDrop software [45]. Standard 90-pin DMF chips are made by Sci-Bots for the DropBot DB3-120. The DMF chips have a two-plate configuration with a $180 \mu \mathrm{m}$ gap height. The bottom plate uses a $0.9 \mathrm{~mm}$ soda-lime glass substrate, chrome electrodes, Parylene-C dielectric layer with $6 \mu \mathrm{m}$ thickness, and FluoroPel hydrophobic layer (Cytonix Corporation, Beltsville, MD, USA). The top plate is a $0.7 \mathrm{~mm}$ ITO-coated glass slide with a FluoroPel hydrophobic layer, and the gap spacing between the two plates is $180 \mu \mathrm{m}$. The DMF chip had a symmetric layout with 8 reservoirs and 76 addressable electrodes. The electrodes patterned on the bottom slide of the chip are $2 \mathrm{~mm} \times 2 \mathrm{~mm}$ squares with a zigzag interface between electrodes. As shown in Figure 1, the chip was divided into four test sites. 


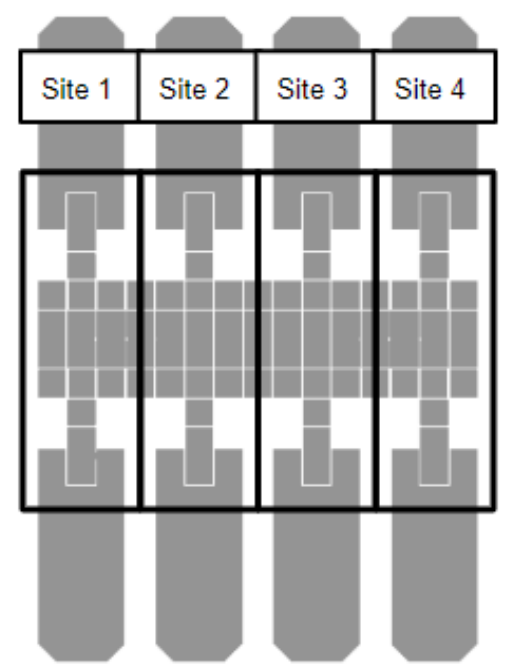

Figure 1. DMF chip electrode layout describing the locations of the test sites.

\subsection{Sample Preparation}

All blood acquired was compliant with the approved F16134 San Jose State Institutional Review Board (IRB) protocol. All fluid specimens were dispensed using a Fisherbrand Finnpipette II $(0.5-10 \mu \mathrm{L})$ with disposable Fisherbrand Micro Pipet tips. All liquid specimens actuated on the DMF platform had an addition of $0.1 \%$ ethylenediamine tetrakis (ethoxylate-block-propoxylate) tetrol (Tetronic 90R4, MilliporeSigma, Burlington, MA). The volume dispensed by pipette has a resolution of $0.1 \mu \mathrm{L}$.

\subsubsection{PBS}

PBS specimens were created from PBS with $0.2 \%$ added surfactant. As the surfactant was very viscous, adding such small amounts to the solution presented a challenge. To create specimens, the surfactant ethylenediamine tetrakis (ethoxylate-block-propoxylate) tetrol (Tetronic 90R4, MilliporeSigma, Burlington, MA) was dispensed into a microcentrifuge tube, using a scale to record weight. Using the recorded weight, PBS was 
dispensed into the microcentrifuge tube by pipette, then mixed using a vortex mixer at $3000 \mathrm{rpm}$ for $15 \mathrm{~s}$. PBS specimens were dyed with fluorescein (MilliporeSigma). To dye, fluorescein was added by pipette to PBS at 1:20 by volume and mixed using a vortex mixer at $3000 \mathrm{rpm}$ for $15 \mathrm{~s}$.

\subsubsection{Whole Blood}

Whole blood specimens were made from whole blood diluted 1:40 in PBS with $0.2 \%$ surfactant. To create specimens, ethylenediamine tetrakis (ethoxylate-block-propoxylate) tetrol was dispensed into a microcentrifuge tube, using a scale to record weight. Using the recorded weight, PBS was dispensed into the microcentrifuge tube by pipette, then mixed using a vortex mixer for 250 relative centrifugal force (RCF) for $20 \mathrm{~min}$. Finally, whole blood was dispensed by the pipette into the microcentrifuge tube and mixed by pipette.

\subsubsection{RBC Suspensions}

RBC specimens were made from RBCs diluted in PBS with $0.2 \%$ surfactant. To separate RBCs from whole blood, blood specimens were spun in a centrifuge set to a force of 250 RCF for $20 \mathrm{~min}$. Plasma and white blood cell layers were removed by pipette. To wash RBCs, $100 \mu \mathrm{L}$ of RBCs was added to $1 \mathrm{~mL}$ of PBS, then spun in a centrifuge set to a force of 250 times gravity for $20 \mathrm{~min}$. The PBS was removed by pipette. All RBCs were washed twice and then diluted to specification in PBS. To create specimens, ethylenediamine tetrakis (ethoxylate-block-propoxylate) tetrol (Tetronic 90R4, MilliporeSigma, Burlington, MA) was dispensed into a microcentrifuge tube, using a scale to record weight. Using the recorded weight, PBS was dispensed into the 
microcentrifuge tube by pipette, then mixed using a vortex mixer. Finally, the washed RBCs were dispensed by pipette into the microcentrifuge tube and mixed by pipette.

\subsubsection{Neuraminidase Treatment}

RBCs were treated with neuraminidase to remove surface charge. First, the cells were separated from whole blood and washed. Next, neuraminidase (MilliporeSigma) was added at a dosage of $100 \mathrm{mU} / \mathrm{mL}$, to the $\mathrm{RBC}$ suspension. For neuraminidase stock of 2 $\mathrm{U} / \mathrm{mL}, 1 \mu \mathrm{L}$ of neuraminidase stock was added for every $20 \mu \mathrm{L}$ of $\mathrm{RBC}$ suspension. The suspension was mixed by pipette and incubated in a water bath for $30 \mathrm{~min}$ at $37^{\circ} \mathrm{C}$. Finally, the cells were washed twice by adding PBS and centrifuging to remove the neuraminidase. The resulting RBC suspension was then used to create rheometry and tensile test solutions as described.

\subsection{Experimental Protocol}

Experimental tensile tests on all specimens were performed on the DropBot DMF platform. Each test was performed on an unused site in order to avoid biofouling. Tensile tests were conducted on designated sites on the EWOD chip as shown in Figure 1. As each chip had eight reservoirs and four test sites, four tensile tests could be conducted simultaneously on a chip. Each tensile test began by applying the prescribed voltage to the respective reservoir. Each of the four reservoirs received $5.5 \mu \mathrm{L}$ of liquid specimen

dispensed by pipette. Next, all specimens were actuated into the test position as shown in Figure 2 from the reservoir using an actuation voltage between $80 \mathrm{~V}$ and $100 \mathrm{~V}$. Finally, 
the specimens were put under tension by activating electrodes as seen in Figure 2 at 100 $\mathrm{V}_{\mathrm{rms}}$ and $10 \mathrm{kHz}$, maintaining voltage until the specimens split or reached equilibrium.

\begin{tabular}{|c|c|c|c|c|}
\hline Step & 1 & 2 & 3 & 4 \\
\hline \multirow{9}{*}{$\begin{array}{l}\text { Electrodes } \\
\text { actuated }\end{array}$} & R0 & R0 & R0 & R0 \\
\hline & B & B & B & B \\
\hline & C & C & C & C \\
\hline & D & D & \multirow{2}{*}{ D } & D \\
\hline & $\mathrm{E}$ & $\mathrm{E}$ & & $\mathrm{E}$ \\
\hline & $\mathrm{F}$ & $\mathrm{F}$ & $\mathrm{E}$ & $\mathrm{F}$ \\
\hline & G & G & $\mathrm{F}$ & $\mathrm{G}$ \\
\hline & $\mathrm{H}$ & $\mathrm{H}$ & H & $\mathrm{H}$ \\
\hline & R1 & R1 & RI & R1 \\
\hline
\end{tabular}

Figure 2. Electrode sequence for actuating liquid specimens into the necessary position for a tensile test. Each lettered region represents an electrode, with shaded regions indicating an activated electrode. 


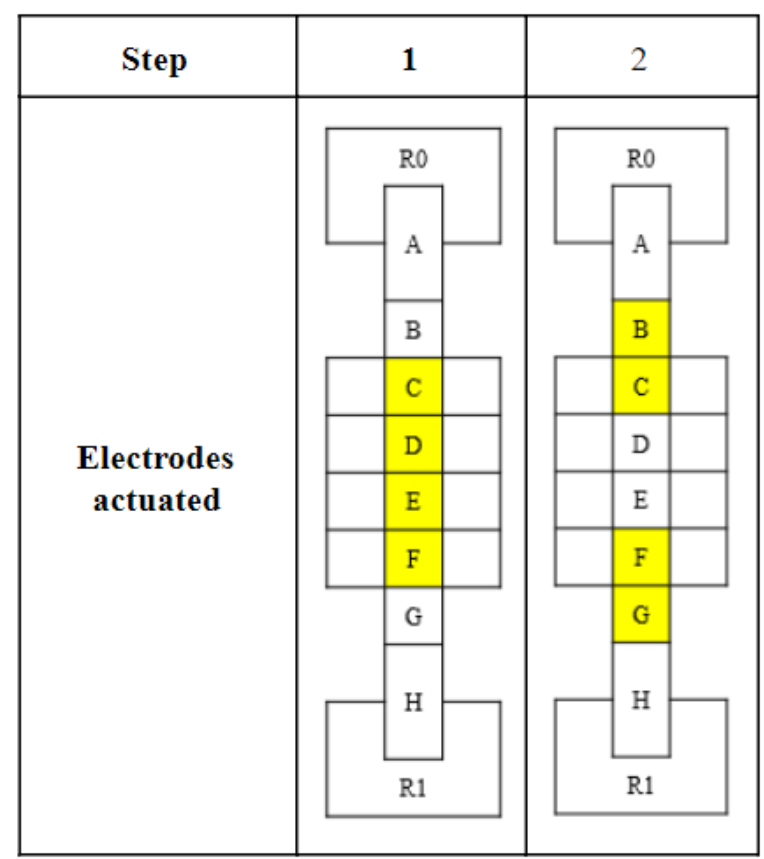

Figure 3. Electrode sequence for tensile test on a test site. Each lettered region represents an electrode, with shaded regions indicating an activated electrode.

\subsubsection{PBS Tensile Tests}

Tensile tests on PBS specimens were performed on the DropBot, ensuring an untouched chip was used to avoid effects of biofouling. Approximately $5 \mu \mathrm{L}$ of PBS specimen was dispensed into an activated reservoir on each test site. For fluorescent imaging, a light shield was placed over the DropBot prior to specimen actuation. Specimens were actuated into position as per Figure 2 and tensile tested as per Figure 3. As described in Appendix 1, PBS specimens were tested on two chips, with tests conducted on four sites on each chip. 


\subsubsection{RBC and Whole Blood Tensile Tests}

Tensile tests on RBC and whole blood specimens were performed on the DropBot, ensuring an untouched chip was used to avoid effects of biofouling [31]. Approximately $5 \mu \mathrm{L}$ of each liquid specimen was dispensed into an activated reservoir on each test site. Specimens were actuated into position as per Figure 2 and tensile tested as per Figure 3. Whole blood tensile tests were conducted on four sites one one chip. RBC tensile tests were conducted using blood drawn from two donors. Neuraminidase-treated RBC suspensions were tested over two chips and untreated RBC suspensions were tested over two separate chips. Tests were conducted on four unique site on each chip, ensuring cells from each donor were tested on both chips.

\subsubsection{Rheometry}

Oscillatory rheometry experiments were performed on neuraminidase-treated RBCs to examine the rheological effects of the treatment. An oscillatory viscometer (Anton Paar MCR 302, Anton Paar GmbH, Graz, Austria) was used to measure the loss modulus against shear for neuraminidase-treated $\mathrm{RBC}$ suspension and untreated $\mathrm{RBC}$ suspension. To prepare suspensions, whole blood was spun in a centrifuge for $20 \mathrm{~min}$ at 250 times the force of gravity to separate RBC, plasma, and leukocyte layers. The plasma and leukocyte layers were extracted and the RBCs were washed twice by creating a solution of $10 \%$ RBCs suspended in PBS, centrifuging, and removing supernatant by pipette. From the washed $\mathrm{RBC}$ suspension, $1 \mathrm{~mL}$ of cells was extracted and treated with neuraminidase. Experiments were performed on cell solutions with (platelet-rich) plasma. 
Suspensions were $10 \%$ RBCs with $50 \%$ PBS and $40 \%$ plasma by volume, and $10 \%$ RBCs with $90 \%$ plasma by volume. The justification for observing solutions with these differing concentrations of plasma is that in the absence of plasma, Jan and Chien [33] observed that the difference between neuraminidase-treated RBCs and untreated RBCs, both suspended in saline, was not discernible until a polymer solution (Dextrans 20, 40, and 80) were added. Wen et al. [34] conducted a similar experiment with rabbit red blood cells suspended in plasma at $\mathrm{H}=35 \%$. In this experiment, both suspensions were run with both treated and untreated cells. An amplitude sweep was performed over a range of strains from $0.01 \%$ to $500 \%$ at an angular frequency of $10 \mathrm{rad} / \mathrm{s}$. A parallel plate measuring system with diameter $15 \mathrm{~mm}$ was used. 


\section{RESULTS AND DISCUSSION}

The blood specimens were subjected to tension on the EWOD-based DMF platform. Figure 4 shows an example of the time progression of necking over the duration of a tensile test. As Figure 4 shows, the profile had an effective neck width of the electrode width and an infinite neck radius prior to actuation. As the electrodes were activated, liquid moves towards the activated electrodes, causing necking over the two deactivated electrodes.

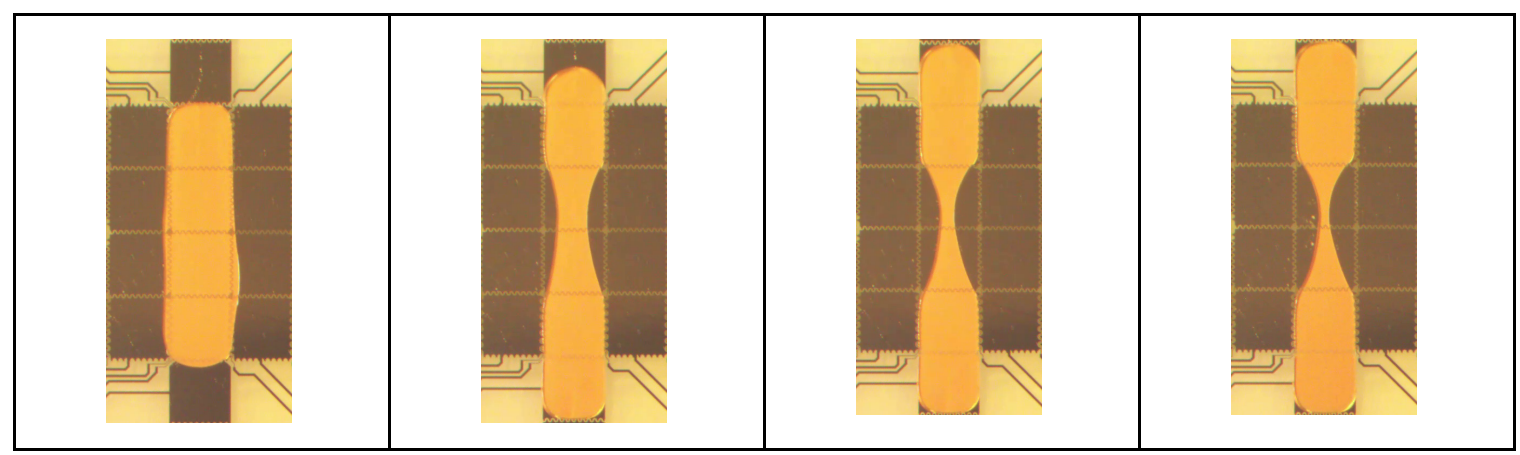

Figure 4. Example of the time progression of necking during an on-chip tensile test. The specimen pictured is whole blood diluted 1:20 in PBS.

\subsection{Parameter Extraction}

Due to high variability in the time development of the necking profile between sites on a DMF chip, a time-invariant deformation parameter is valuable. The parameters measured to describe the necking profile were the minimum neck width $d$ and radius of the necking profile $R$ as illustrated in Figure 5 . 

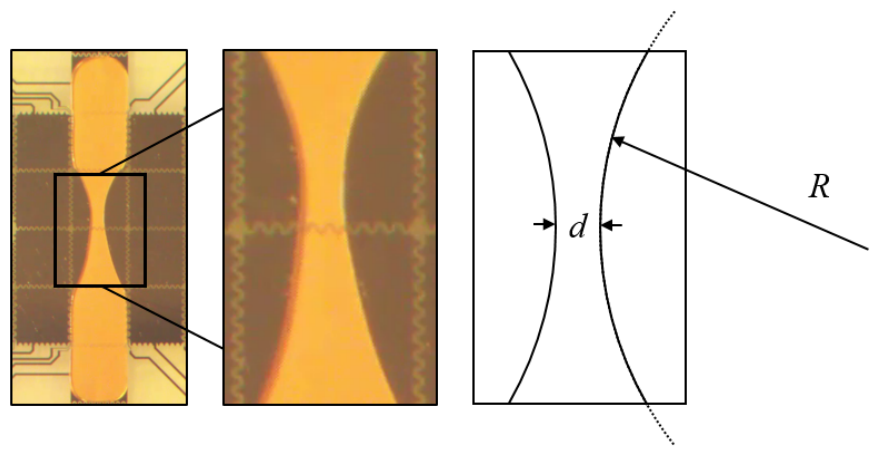

Figure 5. Illustration of necking parameters measured during tests, where $d$ is the minimum neck width, and $R$ is the radius of the necking profile.

Both the neck radius and neck width were obtained from microscope video. Frames from recorded video were extracted at regular intervals using the FFmpeg tool [46]. The resulting images were processed using ImageJ software [47]. First, the frame was cropped to isolate a test site. Next, a color deconvolution [48] was used to separate the pigmentation of the liquid such that the profile can be extracted. The image was then noise-reduced and binarized. To obtain the neck radius, the necking profile was extracted from the binarized image as a separate left and right profile. A robust circle fit method [49], [50] was used to fit a circle to both the left and right profile. Due to asymmetry between the left and right sides of the profile, the radius value for each frame was taken as the average of the radius from both sides of the profile. The neck width was obtained by measuring the width of the profile over the entire neck. The smallest measured width for each frame was taken as the neck width value for that frame. 


\subsection{Time Development of the Necking Profile}

Tensile tests were evaluated on the DMF platform for PBS, whole blood, and RBC suspensions. The development of the necking profile was observed by measuring the minimum neck width of each droplet specimen under tension over the progression of the experiment. Figure 6 shows the neck width versus time for pure PBS. Tests were performed on unique sites over two distinct DMF chips. In addition to a high run-to-run variability, the results show a high chip-to-chip variability (runs 1 through 4 were performed on one chip, while runs 5 through 7 were performed on separate chip).

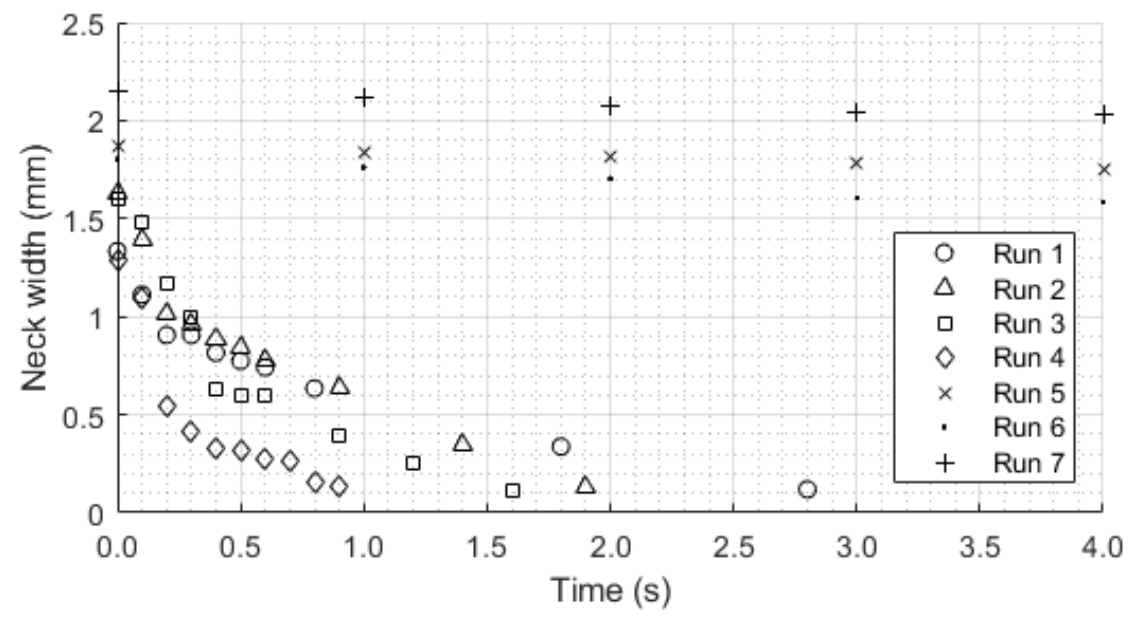

Figure 6. Neck width versus time for tensile tests on PBS.

As shown in Figure 7, tensile test results on whole blood showed similar variation across runs. Whole blood diluted 1:20 in PBS was tested across four sites on one chip. As Figure 7 shows, the necking development slowed dramatically with each run, suggesting the time rate necking development is highly dependent on the on-chip location of the test. 


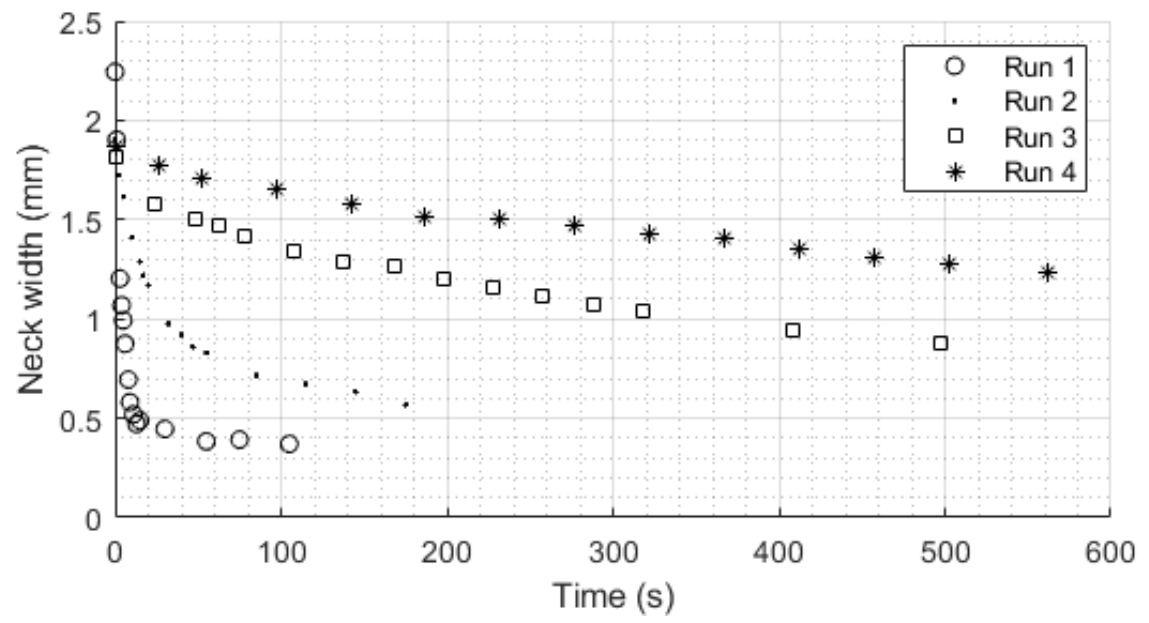

Figure 7. Neck width versus time for tensile tests on whole blood diluted at a 1:20 dilution.

Tensile tests were performed on RBCs collected from two donors. Cells from each donor were tested over multiple distinct lanes on two different chips as in Appendix 1. Figure 8 shows the time development of the necking profile over each run. No significant difference was observed between donor 1 and donor 2. Runs in which the liquid specimen reached equilibrium under tension show a horizontal asymptote (run 1 and run 3) while runs in which the liquid specimen split show a neck width trending to zero (run 2 , run 4 , run 5 , and run 6$)$. 


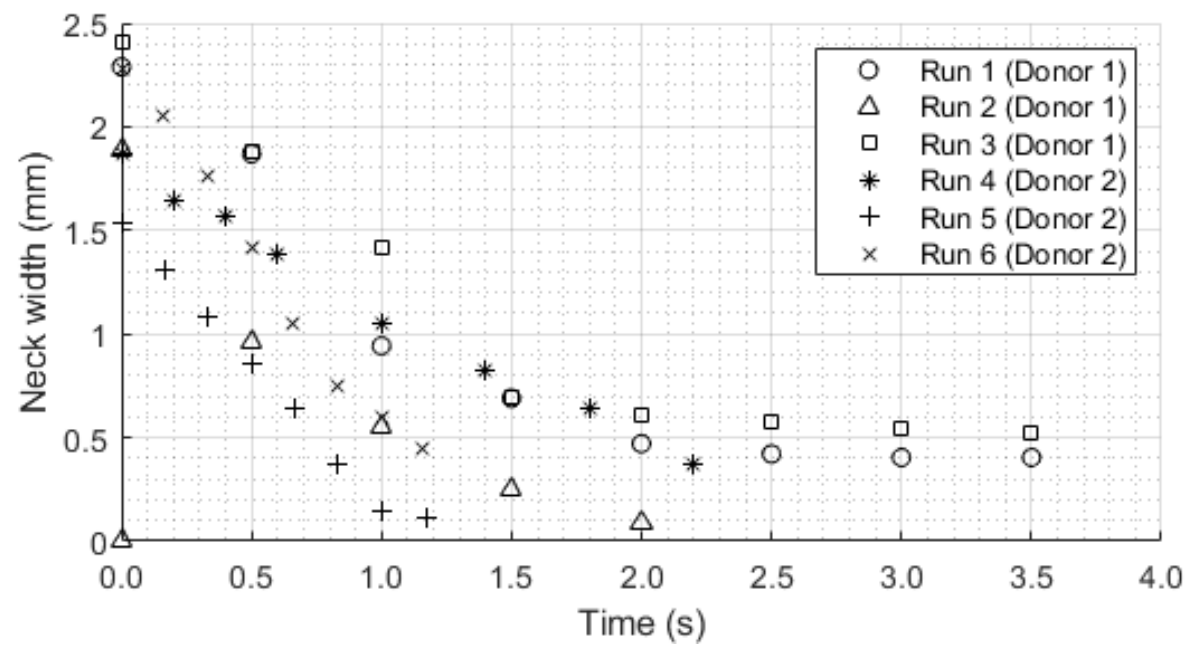

Figure 8. Neck width versus time for tensile tests on $\mathrm{RBC}$ suspensions at $\mathrm{H}=10 \%$.

\subsection{Time-Invariant Development of Necking Profile}

To obtain time-invariant observations of experiments, tensile tests were analyzed using characteristic geometric parameters of the necking profile, neck radius and neck width. A three-parameter exponential model (14) was fitted to experimental data from each experimental substance.

$$
y(x)=C_{1}\left(e^{C_{2} x}-1\right)+C_{3}
$$

Figure 9 shows the neck radius plotted against neck width for the same set of PBS tensile tests shown in Figure 5. As expected, the neck radius decreased with neck width. The exponential fit matched well with experimental data. Neck radius values were very close for all runs, but spread further apart at neck widths from about $1.25 \mathrm{~mm}$ to $1.5 \mathrm{~mm}$. 


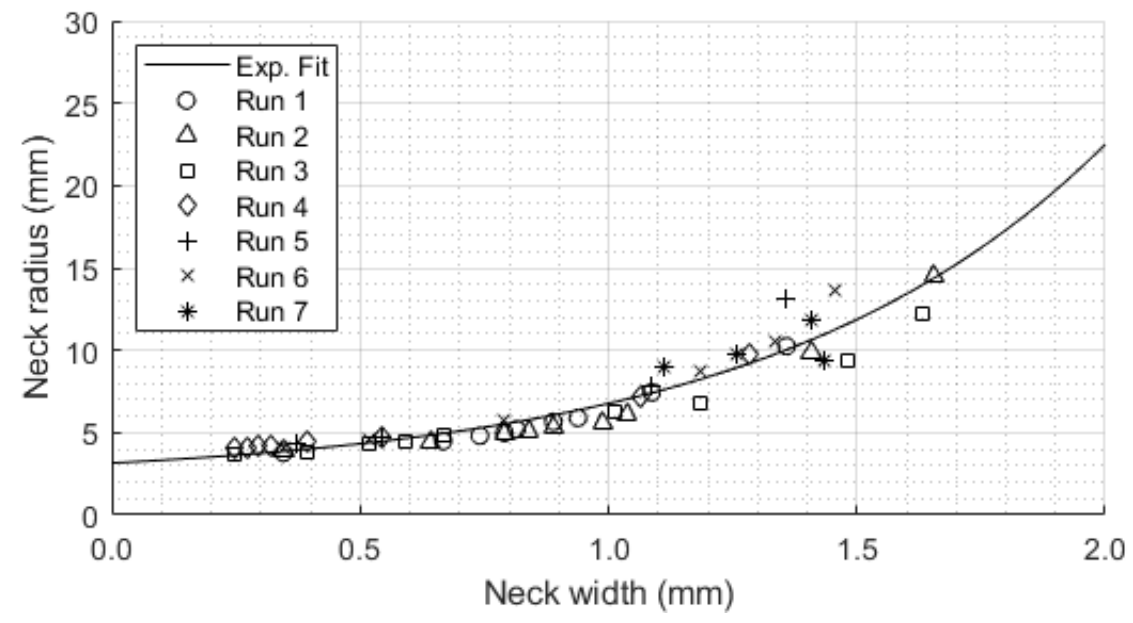

Figure 9. Neck radius versus neck width for tensile tests on PBS.

Whole blood neck radius versus neck width plots are shown in Figure 10 and Figure 11. Neck radius versus neck width measurements for whole blood diluted 1:20 (shown in Figure 10) are from the same tensile test runs as shown in Figure 6. Tensile tests were conducted on whole blood at dilutions of 1:20 (Figure 10) and 1:10 (Figure 11). Figure 11 shows the neck radius decreasing as the neck width decreased, as expected. The neck radius for whole blood diluted 1:20 approached $4.48 \mathrm{~mm}$ at low neck width values. Measurements for each run were close together, and the exponential fit matched the experimental data well. 


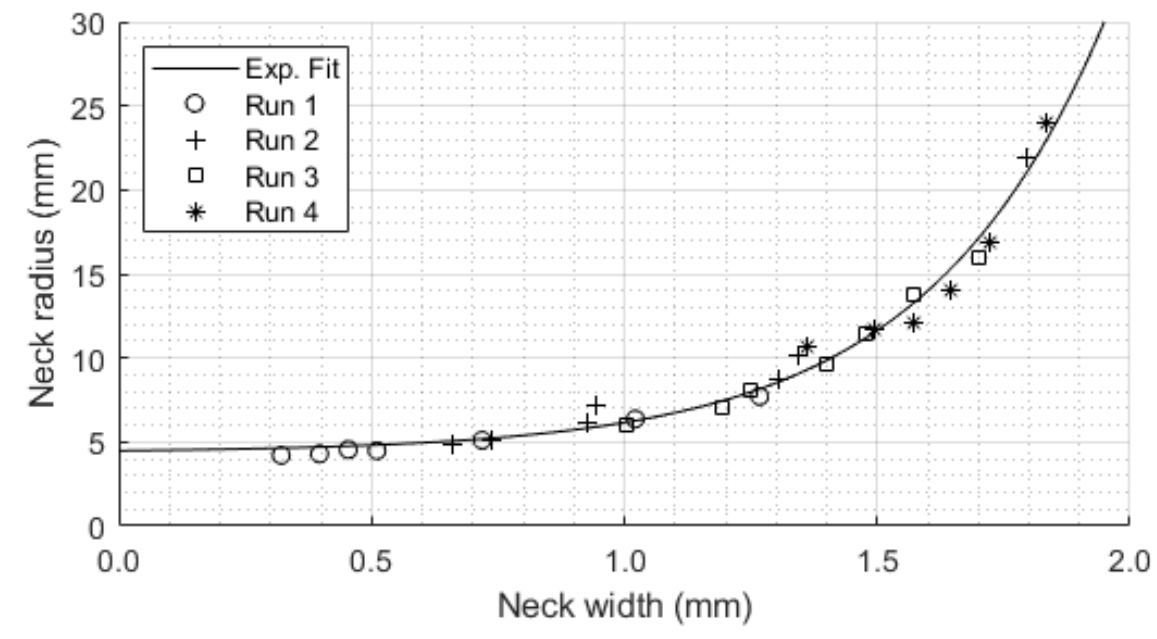

Figure 10. Neck radius versus neck width on whole blood diluted 1:20.

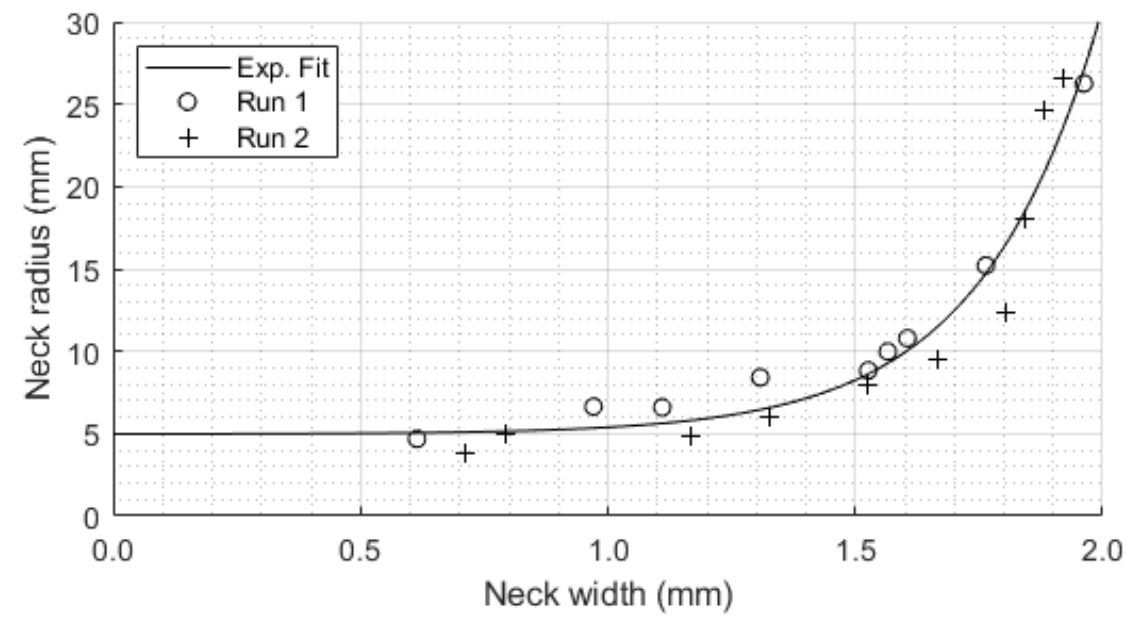

Figure 11. Neck radius versus neck width for tensile tests on whole blood diluted 1:10.

Figure 11 shows the neck radius versus neck width for whole blood diluted 1:10.

Similarly to PBS and whole blood 1:20, measurements between runs are close together.

The exponential fit also matches the data well. Compared to whole blood 1:20, whole blood 1:10 saw a steeper rise in neck radius as neck width increased. The parameters from the exponential fit can be compared from Table 1. For whole blood 1:20, $C_{2}=2.81$ 
$\mathrm{mm}^{-1}$ while for whole blood $1: 10, C_{2}=4.13 \mathrm{~mm}^{-1}$. Furthermore, the horizontal asymptote for whole blood 1:10 was at a neck radius of $4.97 \mathrm{~mm}$. This was slightly larger than the horizontal asymptote for whole blood 1:20, at a neck radius of $4.48 \mathrm{~mm}$. This represents the neck radius to which the liquid specimen approaches as the neck width approaches 0 $\mathrm{mm}$ and the specimen splits.

Figure 12 and Figure 13 show neck radius versus neck width results for RBC suspensions with hematocrit $\mathrm{H}=10 \%$. Figure 13 shows the results for cells treated with neuraminidase to remove surface charge and Figure 12 shows the results for untreated cells. The results shown in Figure 12 were obtained from the same tensile test runs as shown in Figure 8. In Figure 12, run 1 through run 3 were performed a distinct DMF chip from run 4 through run 6. No dependency on DMF chip or run is observed in Figure 12 or Figure 13.

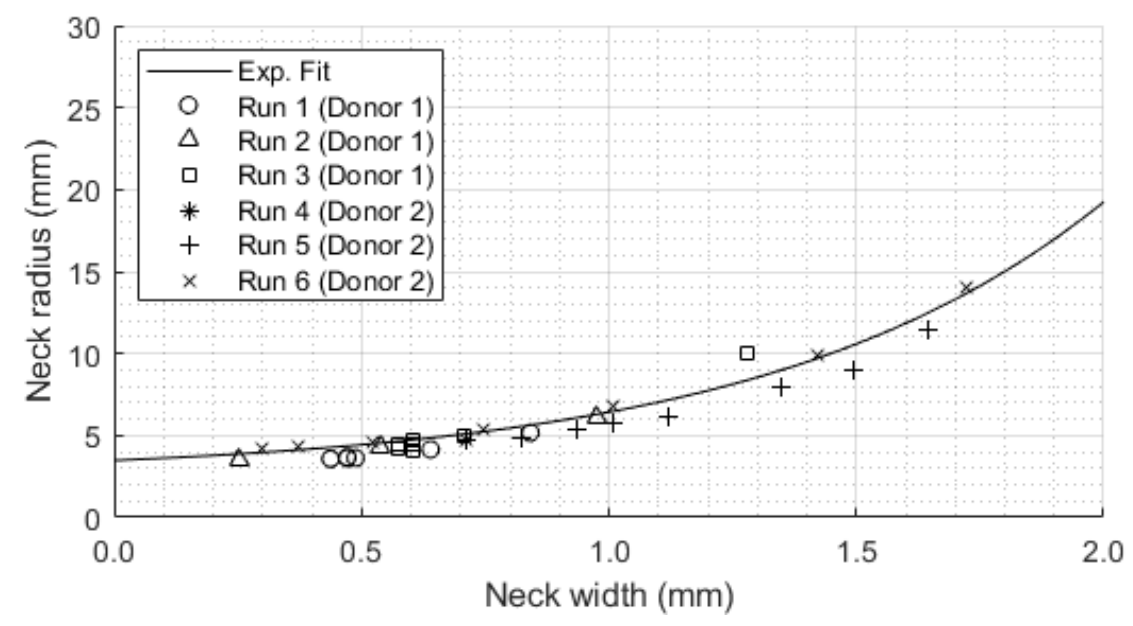

Figure 12. Neck radius versus neck width for tensile tests on RBC suspensions at $\mathrm{H}=10 \%$. 


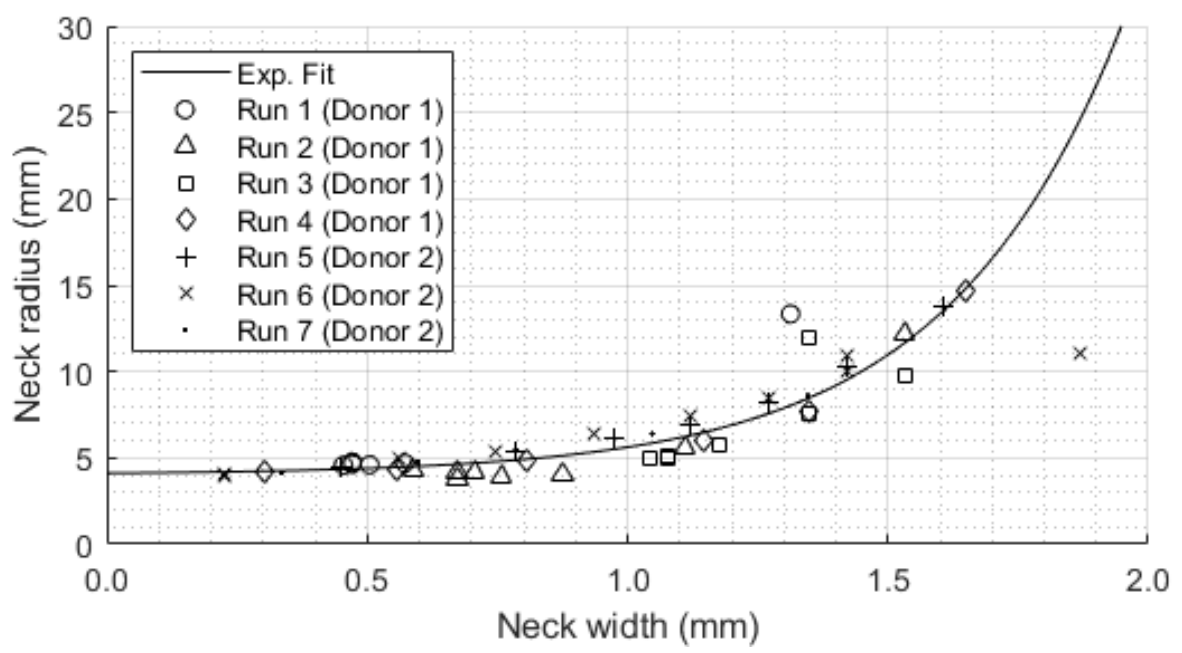

Figure 13. Neck radius versus neck width for tensile tests on neuraminidase-treated RBC suspensions at $\mathrm{H}=10 \%$.

Table 1 reports parameters for exponential fit (14) on all neck radius versus neck width results. Whole blood 1:10 had the smallest value for $C_{1}$ while PBS had the largest value for $C_{1}$. Whole blood 1:10 also had the largest value for the exponential parameter $C_{2}$, while PBS and untreated RBCs had the smallest value for $C_{2}$. Compared to whole blood 1:20, whole blood 1:10 had a lower value for $C_{1}$ and higher values for $C_{2}$ and $C_{3}$. Similarly, neuraminidase-treated RBCs had a lower value for $C_{1}$ and higher values for $C_{2}$ and $C_{3}$ in comparison to untreated RBCs. Of all materials tested, PBS had the highest value of $C_{1}$ and lowest values of $C_{2}$ and $C_{3}$.

Table 1. Parameter Values for Exponential Fits to Tensile Tests.

\begin{tabular}{|c|c|c|c|c|c|}
\hline Parameter & PBS & $\begin{array}{c}\text { Whole } \\
\text { Blood 1:20 }\end{array}$ & $\begin{array}{c}\text { Whole } \\
\text { Blood 1:10 }\end{array}$ & $\begin{array}{c}\text { Untreated } \\
\text { RBCs }\end{array}$ & $\begin{array}{c}\text { Neuraminidase- } \\
\text { Treated RBCs }\end{array}$ \\
\hline$C_{1}[\mathrm{~mm}]$ & 1.08 & 0.108 & 0.00670 & 0.880 & 0.0840 \\
\hline$C_{2}\left[\mathrm{~mm}^{-1}\right]$ & 1.47 & 2.81 & 4.13 & 1.47 & 2.94 \\
\hline$C_{3}[\mathrm{~mm}]$ & 3.15 & 4.48 & 4.97 & 3.48 & 4.11 \\
\hline
\end{tabular}




\subsection{Neuraminidase Treatment of Red Blood Cells}

The treatment of RBCs with neuraminidase is intended to remove the cells' negative surface charge by cleaving sialic acid from the surface of the cells [16]. Rheometry experiments and microscope images were used to observe the effects of neuraminidase treatment on the rheology of RBC suspensions. RBCs were suspended in two solutions with varying amounts of plasma as described. Figure 14 shows microscope images comparing images of untreated and neuraminidase-treated cells suspended in plasma. Both images showed rouleaux formation, with the neuraminidase-treated cells having slightly larger rouleaux stacks. No large differences in cell aggregation were observed.
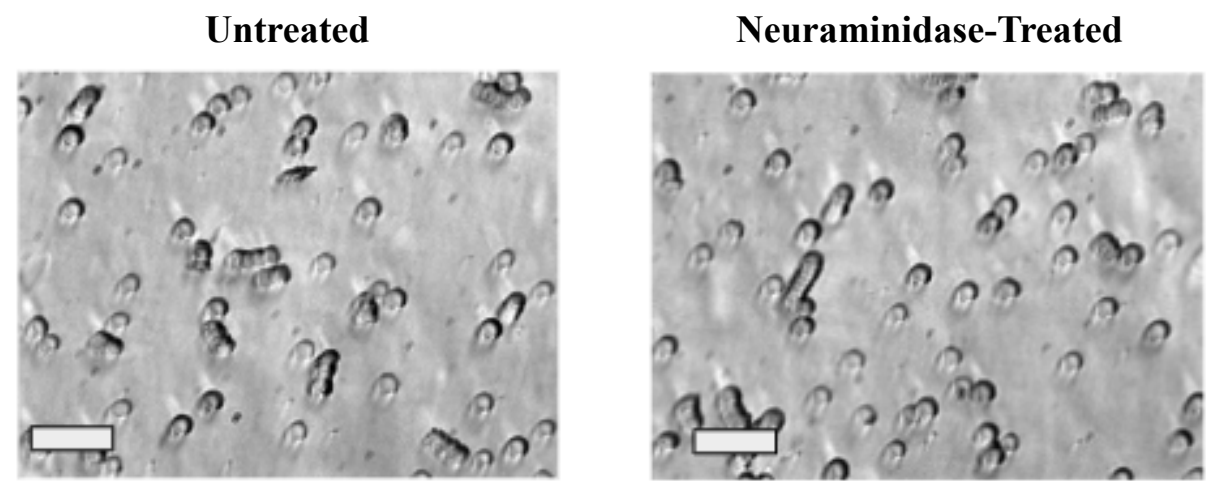

Figure 14. Untreated and neuraminidase-treated RBC suspensions in plasma with $\mathrm{H}=10 \%$. The scale bar in the bottom left corner of each image is $25 \mu \mathrm{m}$ long. 
Untreated

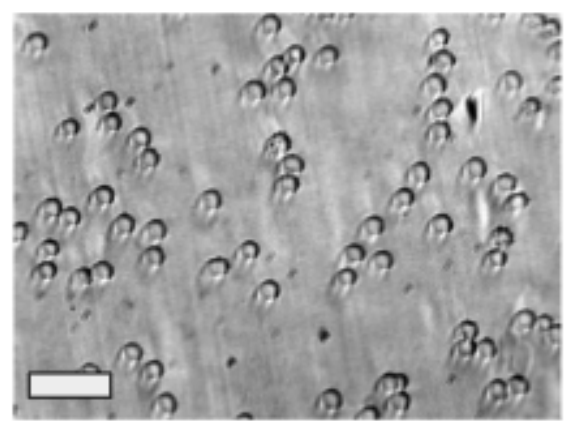

Neuraminidase-Treated

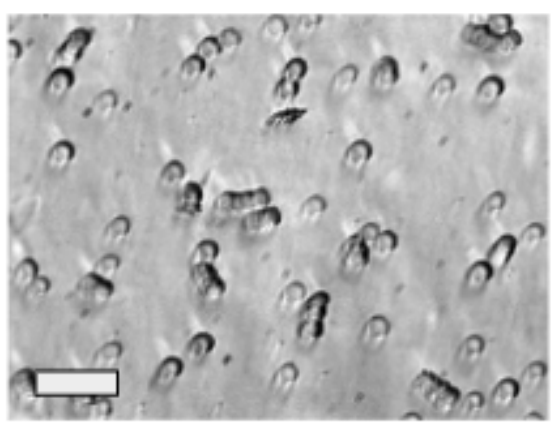

Figure 15. Untreated and neuraminidase-treated RBC suspensions of $40 \%$ plasma, $50 \%$ PBS, and $10 \%$ cells by volume. The scale bar in the bottom left corner of each image is $25 \mu \mathrm{m}$ long.

Figure 15 shows microscope images comparing images of untreated and neuraminidase-treated cells in a suspension of plasma and $\mathrm{PBS}$ at $\mathrm{H}=10 \%$. The solution was $50 \%$ PBS, $40 \%$ plasma, and $10 \%$ cells by volume. No rouleaux formation was observed in the untreated cells while rouleaux formation was observed in the neuraminidase-treated cells. The increased presence of cell aggregates in the neuraminidase-treated cells demonstrates the effect of the treatment, which is expected to increase cell agglutination [16].

Rheometry experiments were conducted on an oscillatory parallel plate rheometer (Anton Paar $\mathrm{GmbH}$ ) using a $15 \mathrm{~mm}$ parallel plate measuring system. Tests were conducted on $\mathrm{RBC}$ suspensions at $\mathrm{H}=10 \%$. Solutions were $50 \% \mathrm{PBS}, 40 \%$ plasma, and $10 \%$ cells by volume, and cells were tested from two separate donors. An amplitude sweep was performed over applied strain values ranging from $0.01 \%$ to $500 \%$, measuring peak shear stress. The angular frequency was $10 \mathrm{rad} / \mathrm{s}$ for all experiments. The difference between shear stress in untreated and neuraminidase-treated specimens over $1 \%$ to $300 \%$ 
shear strain is shown in Figure 16. As shown in Figure 16, the neuraminidase-treated cells had a larger shear stress response compared to untreated cells over the interval of shear strain values.

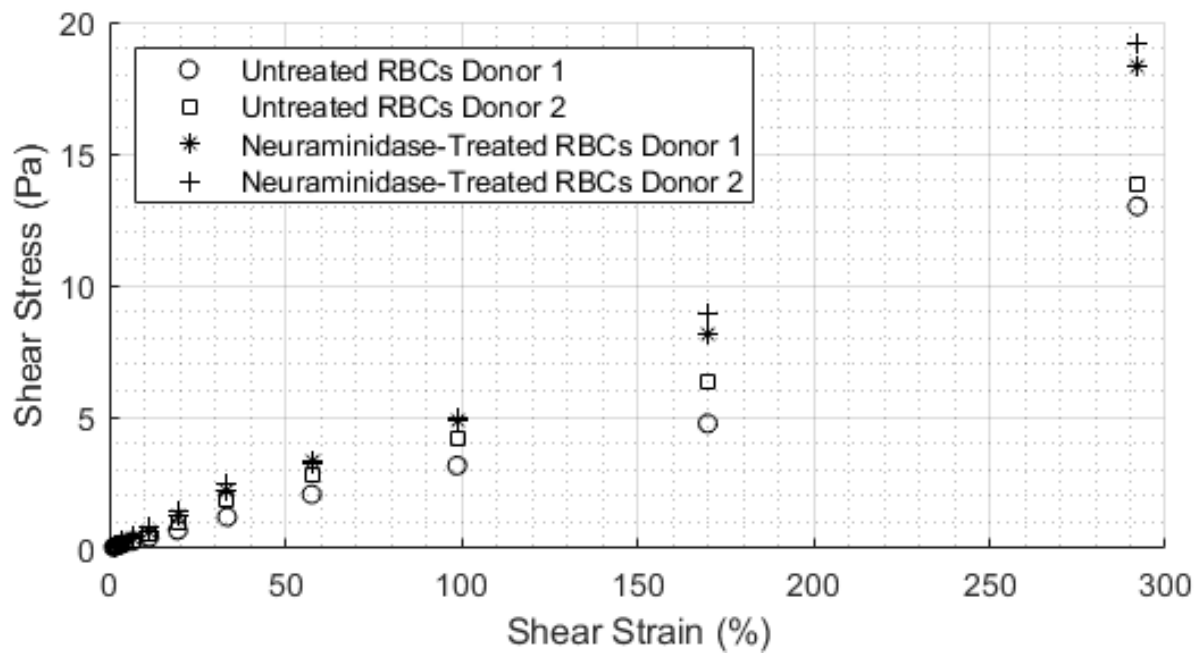

Figure 16. Peak shear stress versus shear strain for RBC suspensions treated with neuraminidase and untreated $\mathrm{RBC}$ suspensions.

The result of the rheometry experiments demonstrate an increased shear stress response to applied shear strain in neuraminidase-treated RBCs in comparison to untreated RBCs. This change in the rheology of cells is further shown by Figure 15, which shows the increase in cell aggregation in neuraminidase-treated RBCs.

\subsection{Estimation of Electrowetting Force}

The force acting on liquid specimens can be estimated by the electrowetting force acting on the droplet. The electrowetting force acts along the contact line between the liquid and electrode boundary, so the electrowetting force was estimated at maximum contact between the liquid and electrode. The line force $f_{w}$ can be calculated from 
equation (3) in terms of the contact angle change, and integrating $f_{w}$ over the maximum contact line per equation (4) results in the total electrowetting force acting on the liquid (5). To determine an estimation of the electrowetting force on whole blood, determining the surface tension of blood is necessary. The surface tension $\gamma$ of whole blood can be described by a linear relationship with temperature $T[51]$.

$$
\gamma(T)=[-0.473 T+70.105] \times 10^{-3} \mathrm{~N} / \mathrm{m}
$$

Per Li et al., the estimated contact angle change for an applied voltage of $100 \mathrm{~V}$ is $23.5^{\circ}$ [19]. Using these values with equation (5) results in a maximum electrowetting force of $45.8 \mu \mathrm{N}$ acting on a blood droplet specimen when $100 \mathrm{~V}$ is applied.

\subsection{Discussion}

The time results of tensile tests showed higher variability as well as dependence on DMF chip and site in comparison to time-invariant results. This was shown to occur in PBS, whole blood, and RBC suspensions. Furthermore, whole blood and RBC specimens were observed to separate by component during testing on DMF chips. This phenomenon was investigated using the color profile of the neck during tensile tests on RBC suspensions.

\subsubsection{Variability in Tensile Behavior}

Evaluating the neck width against time over the duration of a tensile test on a DMF platform results in a profile which showed a high dependence on the site and chip on which the test was run. Particularly for whole blood tensile tests, the duration of a tensile test ranged from seconds to dozens of minutes. In each case, this could be due to several 
factors. One such factor is local microtopography of hydrophobic coatings on the top and bottom plate of the chip, which can be very influential on the droplet behavior [52]. Another factor is the parallelism between the top and bottom plate of the chip, which varies the gap spacing between plates. This highly affects the capacitance (3) of the circuit made by the electrodes, dielectric layer, and liquid.

The time-invariant method produced results with no observed dependence on test site or chip. In addition, the development of geometric parameters was far closer between runs. This gives the time-invariant method of observation on a DMF platform value in comparison to time measurement, as it removes the high time variability. An exponential fit with three parameters (14) matched measured data well for PBS, whole blood, and RBC suspension tensile tests. Whole blood diluted 1:10 experienced a steeper exponential increase, with a $C_{2}$ value of $4.13 \mathrm{~mm}^{-1}$, compared to whole blood diluted 1:20, with a $C_{2}$ value of $2.81 \mathrm{~mm}^{-1}$. Additionally, whole blood 1:10 had a smaller $C_{1}$ value and larger $C_{3}$ value than whole blood 1:20. Following this trend, PBS had a larger $C_{1}$ value, smaller $C_{2}$ value, and smaller $C_{3}$ value. This indicates that the concentration of blood in a liquid specimen tested on a DMF device influences the development of the necking profile, and has potential for developing further testing methods.

Tensile tests on RBC suspensions did not see any dependence on donor. The untreated RBC suspensions had a higher $C_{1}$ value, a lower $C_{2}$ value, and a lower $C_{3}$ value compared to neuraminidase-treated RBC suspensions. This suggests that the untreated cells behaved closer to pure PBS, while the treated cells behaved closer to whole blood. The 
neuraminidase treatment was observed in Figure 14 to slightly increase the cells' tendency to aggregate, and Figure 15 demonstrates the effect on the rheology of RBC suspensions. The rheometry results in addition to the increased agglutinability observed in neuraminidase-treated RBCs show that the altered rheology of treated RBCs affected the development of the necking profile during tensile tests. The increased agglutinability of cells has been shown to increase viscosity within a suspension [16], [34], [53]. The increased viscosity in treated $\mathrm{RBC}$ suspensions is consistent with a larger minimum neck radius in comparison to untreated $\mathrm{RBC}$ suspensions, quantified by $C_{3}$ in the exponential fit. The influence of viscosity between untreated and treated RBCs suggests that the comparison between dilutions of whole blood are also influenced by viscosity, as the exponential parameter comparison follows the same pattern.

Notable is the varied behavior of different substances on a DMF platform. RBC suspensions and PBS exhibited the fastest response to electrowetting actuation, followed by whole blood and fresh-frozen plasma (FFP). FFP was used exclusively during preliminary tests over the course of this study, but proved highly difficult to actuate consistently on-chip, often requiring high actuation voltages for a response. Additionally, FFP was observed to gradually change from transparent to opaque and reduce response while under applied electrowetting over several minutes. While several tensile experimental protocols were developed for FFP, the challenges of reliably transporting FFP specimens to test sites on-chip caused these experiments to be impractical. 


\subsubsection{Component Separation}

Observed in several tensile tests was the separation of components within whole blood and RBC suspensions. Figure 17 shows an example of this separation which occurred in whole blood diluted 1:10 on repeat tensile testing. This is likely due to the differences in response of RBCs, plasma, and PBS to the applied electrowetting forces.
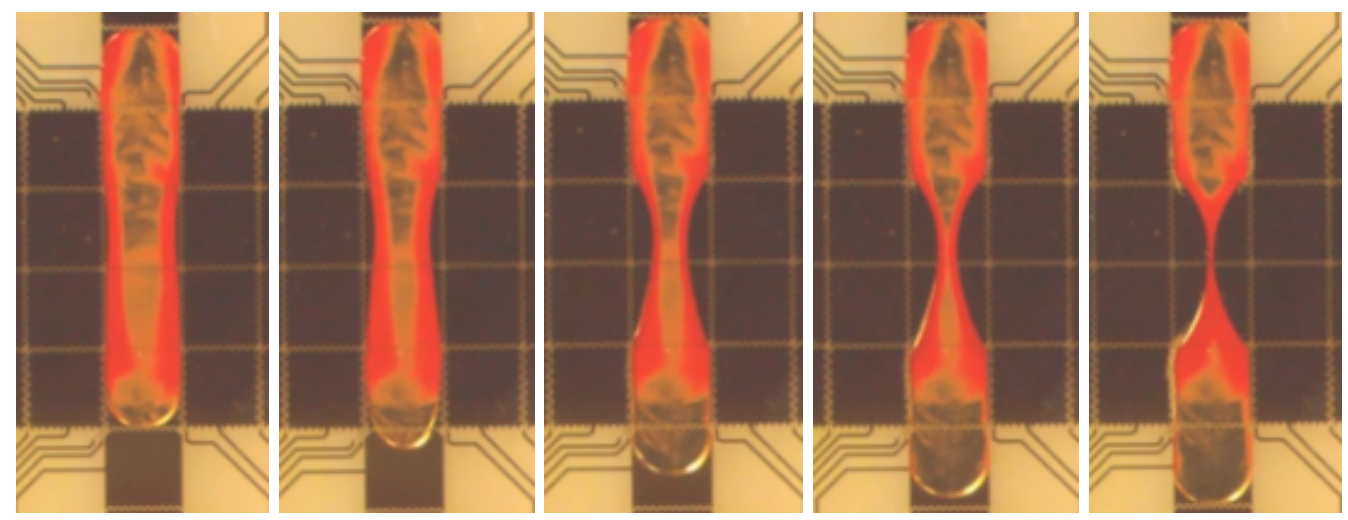

Figure 17. Development of component separation during tensile testing on whole blood diluted 1:10.

Additionally, this separation of components can plausibly explain the difficulties of actuating whole blood relative to PBS and RBC suspensions. Whole blood includes plasma, which had a low response to electrowetting forces, but also contains RBCs, and is then diluted in PBS. This effect offers further potential to analyze the components of blood and their behavior within an applied electric field.

The separation between components was further observed in additional tensile tests performed on untreated and neuraminidase-treated RBC suspensions. Due to the fast response to tensile force in both the treated and untreated RBC suspensions, testing was conducted by performing two consecutive tensile tests on each suspension on the same 
site, with $30 \mathrm{~s}$ between each test. Figure 18 shows the separation in untreated RBCs at each tensile test by showing the suspension at the same neck width for both tests. The normalized level for the red channel of the captured RGB (red, green, and blue) image remained near 1.0 for across the neck width the first tensile test, but had a distinct valley centered near the middle of the neck for the second tensile test.

(a)
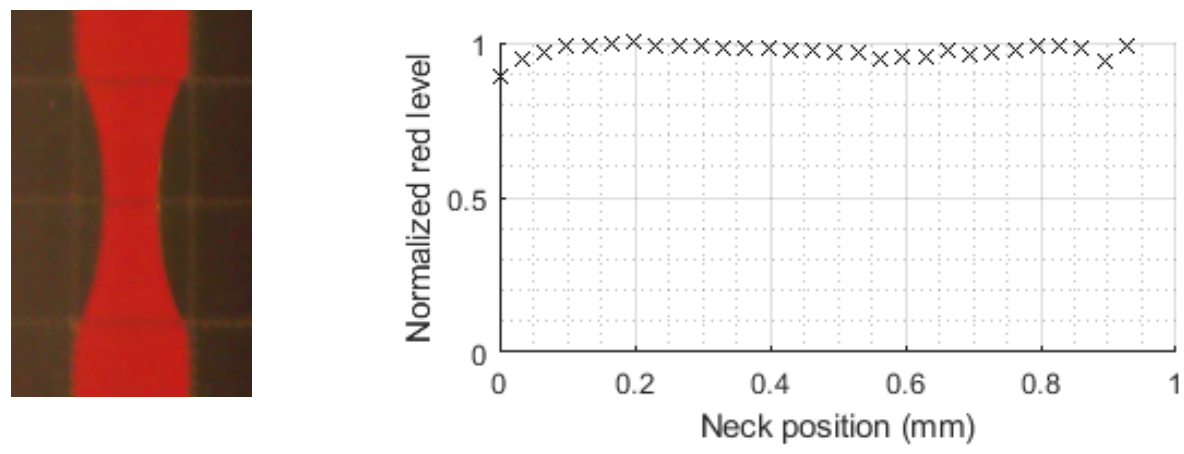

(b)
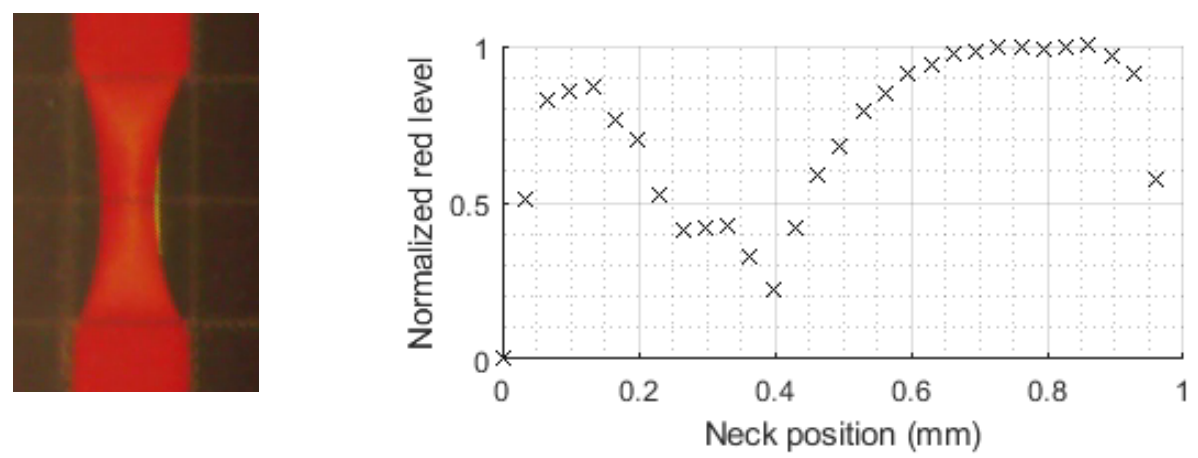

Figure 18. Separation of an untreated $\mathrm{RBC}$ suspension at $\mathrm{H}=10 \%$ over two consecutive tensile tests. The image and plot at (a) and (b) show the suspension during the first and second tensile test, respectively, at the same neck width. The plots show the normalized red level extracted from the image across the minimum neck width.

The neuraminidase-treated RBC suspension experienced a greater separation than the untreated suspension. In Figure 19, which shows the separation of a treated suspension, the change between the consecutive tensile tests is greater than the corresponding images in Figure 18, which shows the separation in an untreated suspension. This separation is 
confirmed in the normalized red level plots for both suspensions. The minimum red level for the separated untreated suspension was 0.21 , while the minimum red level for the separated treated suspension was below 0.10 . The neuraminidase treatment caused an increased cell agglutination, which was confirmed in Figure 14 and Figure 15. A likely factor in the increased separation is the presence of larger cell aggregates in the neuraminidase-treated RBC suspensions. Additionally, a decreased repulsive force between cells increased their tendency to move towards the sides of the neck in the treated suspensions. In the untreated RBC suspensions, the cells retained their regular repulsive force and did not have an increased tendency to aggregate, leading to less separation than in the treated RBC suspensions. 
(a)
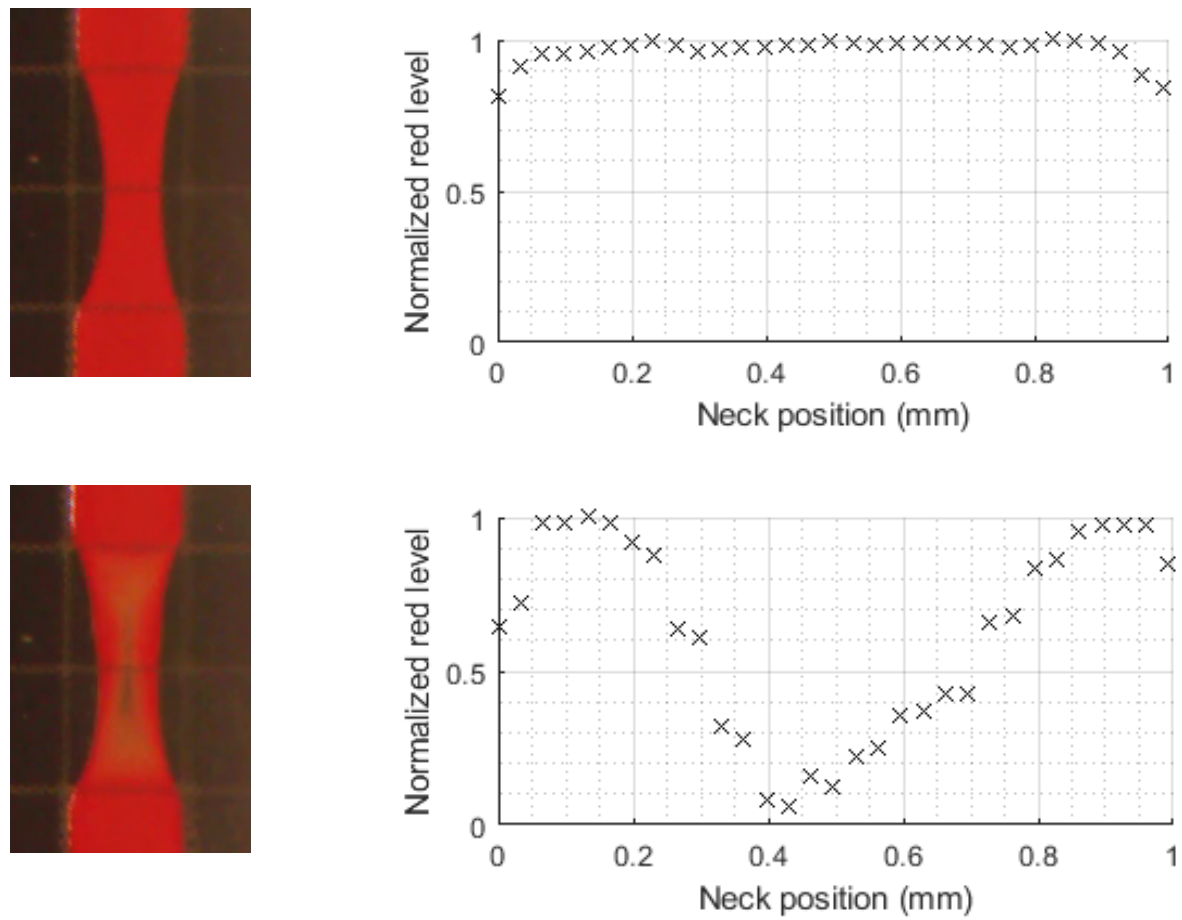

Figure 19. Separation of a neuraminidase-treated $\mathrm{RBC}$ suspension at $\mathrm{H}=10 \%$ over two consecutive tensile tests. The image and plot at (a) and (b) show the suspension during the first and second tensile test at the same neck width. The plots show the normalized red value extracted from the image across the minimum neck width. 


\section{CONCLUSIONS}

A time-invariant method was developed to observe the development of the necking geometry of liquid specimens on an electrowetting DMF platform. This method was based on applying electrowetting force such that the liquid specimen is in tension, and evaluating the neck radius against the neck width over the evolution of the specimen's deformation. Tensile tests were performed on PBS, whole blood diluted 1:20 in PBS, and RBCs suspended in PBS at $\mathrm{H}=10 \%$. The time evolution of liquid necking was evaluated by measuring the neck width against time. For all substances, this was shown to have a high dependence on the site and DMF chip on which the test was performed. The time-invariant method was then used and compared to the time evolution for PBS, whole blood, and RBC specimens. In comparison to the time evolution, the time-invariant data saw dramatically decreased variability between runs as well as no dependence on test site or chip. Due to this, the time-invariant method using the geometry of the necking profile provides a way to evaluate liquid specimens on a DMF platform which mitigates inherent difficulties of electrowetting-based testing, including local topography of layers on DMF chips and parallelism between the top and bottom plate of a two-plate DMF system.

Tensile tests on a DMF platform were used to observe the influence of RBC surface charge on the development of necking. To remove surface charge, RBCs were treated with neuraminidase. Microscope images showed a slight increase in cell aggregates in neuraminidase-treated RBCs, and parallel-plate rheometry showed an increased shear stress response to applied strain. Neck width versus neck radius results were compared 
between treated and untreated RBC suspensions using a three-parameter exponential fit to experimental data. Neuraminidase-treated RBCs had a lower scaling parameter $C_{1}$ value, a higher exponential parameter $C_{2}$ value, and a higher minimum neck radius parameter $C_{3}$ value than untreated RBCs, indicating a more slender aspect ratio over the duration of the tensile tests. This is consistent with neuraminidase-treated RBCs having a greater tendency to aggregate and consequently exhibit higher viscosity. The necking development was also compared between whole blood at different concentrations using the exponential fit to experimental data. Whole blood diluted 1:20 had a higher $C_{l}$ value, a lower $C_{2}$ value, and lower $C_{3}$ value in comparison to whole blood diluted 1:10. This follows the trend of the RBC parameter comparison, with whole blood 1:20 and untreated $\mathrm{RBC}$ suspensions having a lower viscosity than whole blood 1:10 and treated $\mathrm{RBC}$ suspensions having a higher viscosity. These results support the hypothesis that independent of time, a characteristic necking profile exists for whole blood and RBC suspensions on an electrowetting DMF platform. 


\section{REFERENCES CITED}

[1] V. Kuhn et al., "Red Blood Cell Function and Dysfunction: Redox Regulation, Nitric Oxide Metabolism, Anemia," Antioxid. Redox Signal., vol. 26, no. 13, pp. 718-742, May 2017.

[2] T. V. Colace, G. W. Tormoen, O. J. T. McCarty, and S. L. Diamond, "Microfluidics and coagulation biology," Annu. Rev. Biomed. Eng., vol. 15, pp. 283-303, May 2013.

[3] Y.-P. Zhao and Y. Wang, "Fundamentals and Applications of Electrowetting," rev adhes adhesives, vol. 1, no. 1, pp. 114-174, Feb. 2013.

[4] C. Quilliet and B. Berge, "Electrowetting: a recent outbreak," Curr. Opin. Colloid Interface Sci., vol. 6, no. 1, pp. 34-39, Feb. 2001.

[5] G. J. Lippmann, "Relation entre les phénomènes électriques et capillaires," $C$. $R$. Hebd. Seances Acad. Sci., vol. 76, pp. 1407-1408, 1873.

[6] D. Das, S. Das, and K. Biswas, "Effect of electrode geometry on voltage reduction in EWOD based devices," in 2010 International Conference on Systems in Medicine and Biology, 2010, pp. 371-375.

[7] R. Sista et al., "Development of a digital microfluidic platform for point of care testing," Lab Chip, vol. 8, no. 12, p. 2091, 2008.

[8] B. Berge, "Electrocapillarity and wetting of insulator films by water," C.R. Acad. Sci. Ser. IIc: Chim., vol. 317, no. 2, pp. 157-163, 1993.

[9] P. Garcí-Sánchez and F. Mugele, "Fundamentals of Electrowetting and Applications in Microsystems," in Electrokinetics and Electrohydrodynamics in Microsystems, A. Ramos, Ed. Vienna: Springer Vienna, 2011, pp. 85-125.

[10] S. K. Cho, H. Moon, and C.-J. Kim, "Creating, transporting, cutting, and merging liquid droplets by electrowetting-based actuation for digital microfluidic circuits," $J$. Microelectromech. Syst., vol. 12, no. 1, pp. 70-80, Feb. 2003.

[11] H.-H. Shen, S.-K. Fan, C.-J. Kim, and D.-J. Yao, "EWOD microfluidic systems for biomedical applications," Microfluid. Nanofluidics, vol. 16, no. 5, pp. 965-987, May 2014.

[12] H. Ren, V. Srinivasan, and R. B. Fair, "Automated electrowetting-based droplet dispensing with good reproducibility," Micro Total Anal. Syst., pp. 993-996, 2003.

[13] M. Chiang and S. Fan, "Electric manipulations of hydrogel on a digital microfluidic platform," in 2012 7th IEEE International Conference on Nano/Micro Engineered 
and Molecular Systems (NEMS), 2012, pp. 407-410.

[14] G. Weiss and L. T. Goodnough, "Anemia of chronic disease," N. Engl. J. Med., vol. 352, no. 10, pp. 1011-1023, Mar. 2005.

[15] T. Q. Richardson and A. C. Guyton, "Effects of polycythemia and anemia on cardiac output and other circulatory factors," American Journal of Physiology-Legacy Content, vol. 197, no. 6, pp. 1167-1170, Dec. 1959.

[16] S. J. Luner, P. Sturgeon, D. Szklarek, and D. T. McQuiston, "Effects of Proteases and Neuraminidase on RBC Surface Charge and Agglutination," Vox Sang., vol. 28, no. 3, pp. 184-199, Mar. 1975.

[17] P. Paik, V. K. Pamula, and R. B. Fair, "Rapid droplet mixers for digital microfluidic systems," Lab Chip, vol. 3, no. 4, pp. 253-259, Nov. 2003.

[18] I. Barbulovic-Nad, H. Yang, P. S. Park, and A. R. Wheeler, "Digital microfluidics for cell-based assays," Lab Chip, vol. 8, no. 4, pp. 519-526, Apr. 2008.

[19] L. Li, H. Hu, H. Lin, and D.-T. Ye, "Electrowetting of the blood droplet on the hydrophobic film of the EWOD chips," in 2005 IEEE Engineering in Medicine and Biology 27th Annual Conference, 2005, pp. 1941-1944.

[20] M. J. Jebrail et al., "A digital microfluidic method for dried blood spot analysis," Lab Chip, vol. 11, no. 19, pp. 3218-3224, Oct. 2011.

[21] A. H. C. Ng et al., "A digital microfluidic system for serological immunoassays in remote settings," Sci. Transl. Med., vol. 10, no. 438, Apr. 2018.

[22] O. K. Baskurt and H. J. Meiselman, "Blood rheology and hemodynamics," Semin. Thromb. Hemost., vol. 29, no. 5, pp. 435-450, Oct. 2003.

[23] G. B. Thurston, "Viscoelasticity of human blood," Biophys. J., vol. 12, no. 9, pp. 1205-1217, Sep. 1972.

[24] M. W. Rampling, "Red cell aggregation and yield stress," Clinical blood rheology, vol. 1, pp. 45-64, 1988.

[25] H. Schmid-Schönbein, R. E. Wells, and J. Goldstone, "Fluid drop-like behaviour of erythrocytes - disturbance in pathology and its quantification," Biorheology, vol. 7, no. 4, pp. 227-234, 1971.

[26] D. A. Fedosov, W. Pan, B. Caswell, G. Gompper, and G. E. Karniadakis, "Predicting human blood viscosity in silico," Proc. Natl. Acad. Sci. U. S. A., vol. 108, no. 29, pp. 11772-11777, Jul. 2011. 
[27] W. Pan, B. Caswell, and G. E. Karniadakis, "A low-dimensional model for the red blood cell," Soft Matter, vol. 6, no. 18, Sep. 2010.

[28] W. Reinke, P. C. Johnson, and P. Gaehtgens, "Effect of Shear Rate Variation on Apparent Viscosity of Human Blood in Tlibes of 29 to 94 jLim Diameter," Circ. Res., 1986.

[29] S. Gupta, W. S. Wang, and S. A. Vanapalli, "Microfluidic viscometers for shear rheology of complex fluids and biofluids," Biomicrofluidics, vol. 10, no. 4, p. 043402, Jul. 2016.

[30] C. J. Pipe and G. H. McKinley, "Microfluidic rheometry," Mech. Res. Commun., vol. 36, no. 1, pp. 110-120, Jan. 2009.

[31] R. Mukhopadhyay, "When Microfluidic Devices Go Bad," Anal. Chem., vol. 77, no. 21, p. 429 A-432 A, Nov. 2005.

[32] A. G. Banpurkar, M. H. G. Duits, D. van den Ende, and F. Mugele, "Electrowetting of Complex Fluids: Perspectives for Rheometry on Chip," Langmuir, vol. 25, no. 2, pp. 1245-1252, Jan. 2009.

[33] K. M. Jan and S. Chien, "Role of surface electric charge in red blood cell interactions," J. Gen. Physiol., vol. 61, no. 5, pp. 638-654, May 1973.

[34] Z. Wen et al., "Influence of neuraminidase on the characteristics of microrheology of red blood cells," Clin. Hemorheol. Microcirc., vol. 23, no. 1, pp. 51-57, 2000.

[35] J. Matta and R. Tytus, "Liquid stretching using a falling cylinder," J. Non-Newtonian Fluid Mech., vol. 35, no. 2, pp. 215-229, Jan. 1990.

[36] P. Szabo, G. H. McKinley, and C. Clasen, "Constant force extensional rheometry of polymer solutions," J. Non-Newtonian Fluid Mech., vol. 169-170, pp. 26-41, Feb. 2012.

[37] P. C. Sousa, R. Vaz, A. Cerejo, M. S. N. Oliveira, M. A. Alves, and F. T. Pinho, "Rheological behavior of human blood in uniaxial extensional flow," J. Rheol., vol. 62, no. 2, pp. 447-456, Mar. 2018.

[38] V. M. Entov and E. J. Hinch, "Effect of a spectrum of relaxation times on the capillary thinning of a filament of elastic liquid," J. Non-Newtonian Fluid Mech., vol. 72, no. 1, pp. 31-53, Sep. 1997.

[39] K. Choi, A. H. C. Ng, R. Fobel, and A. R. Wheeler, "Digital microfluidics," Annu. Rev. Anal. Chem., vol. 5, pp. 413-440, Apr. 2012.

[40] P. Lambert, Ed., Surface Tension in Microsystems: Engineering Below the Capillary 
Length. 2013.

[41] J. Wu, R. Yue, X. Zeng, and L. Liu, "Droplets actuating chip based on electrowetting-on-dielectric," Front. Electr. Electron. Eng. Chin., vol. 2, no. 3, pp. 345-349, Jul. 2007.

[42] J. Zeng and T. Korsmeyer, "Principles of droplet electrohydrodynamics for lab-on-a-chip," Lab Chip, vol. 4, no. 4, pp. 265-277, Aug. 2004.

[43] T. B. Jones, K. L. Wang, and D. J. Yao, "Frequency-dependent electromechanics of aqueous liquids: electrowetting and dielectrophoresis," Langmuir, vol. 20, no. 7, pp. 2813-2818, Mar. 2004.

[44] D. Chatterjee, H. Shepherd, and R. L. Garrell, "Electromechanical model for actuating liquids in a two-plate droplet microfluidic device," Lab Chip, vol. 9, no. 9, pp. 1219-1229, May 2009.

[45] R. Fobel, C. Fobel, and A. R. Wheeler, "DropBot: An open-source digital microfluidic control system with precise control of electrostatic driving force and instantaneous drop velocity measurement," Appl. Phys. Lett., vol. 102, no. 19, p. 193513, May 2013.

[46] F. Bellard, "Ffmpeg multimedia system," FFmpeg. [Last accessed: November 2015]. https://www. ffmpeg. org/about. html, 2005.

[47] W. S. Rasband, "Imagej, us national institutes of health, bethesda, maryland, usa," http://imagej. nih. gov/ij/, 2011.

[48] A. C. Ruifrok and D. A. Johnston, "Quantification of histochemical staining by color deconvolution," Anal. Quant. Cytol. Histol., vol. 23, no. 4, pp. 291-299, Aug. 2001.

[49] N. Chernov, “Circle Fit (Pratt Method)," MathWorks File Exchange, 10-Jan-2009. [Online]. Available: https://www.mathworks.com/matlabcentral/fileexchange/22643-circle-fit-pratt-meth od. [Accessed: 10-Mar-2019].

[50] V. Pratt, "Direct Least-squares Fitting of Algebraic Surfaces," in Proceedings of the 14th Annual Conference on Computer Graphics and Interactive Techniques, 1987, pp. $145-152$.

[51] J. Rosina, E. Kvasnák, D. Suta, H. Kolárová, J. Málek, and L. Krajci, “Temperature dependence of blood surface tension," Physiol. Res., vol. 56 Suppl 1, pp. S93-8, May 2007.

[52] V. Jain, V. Devarasetty, and R. Patrikar, "Effect of electrode geometry on droplet velocity in open EWOD based device for digital microfluidics applications," $J$. 
Electrostat., vol. 87, pp. 11-18, Jun. 2017.

[53] D. A. Fedosov, B. Caswell, and G. E. Karniadakis, "A multiscale red blood cell model with accurate mechanics, rheology, and dynamics," Biophys. J., vol. 98, no. 10, pp. 2215-2225, May 2010. 


\section{APPENDIX A: Experimental Table}

\begin{tabular}{|c|c|c|c|c|c|}
\hline $\begin{array}{c}\text { Specimen } \\
\text { content }\end{array}$ & Chip & Site 1 & Site 2 & Site 3 & Site 4 \\
\hline PBS & Chip 1 & No Donor & No Donor & No Donor & No Donor \\
\hline PBS & Chip 2 & No Donor & No Donor & No Donor & No Donor \\
\hline Whole Blood 1:20 & Chip 7 & Donor 3 & Donor 3 & Donor 3 & Donor 3 \\
\hline Whole Blood 1:10 & Chip 8 & Donor 4 & Donor 4 & - & - \\
\hline Untreated RBC & Chip 3 & Donor 1 & Donor 2 & Donor 1 & Donor 2 \\
\hline Untreated RBC & Chip 4 & Donor 2 & Donor 1 & Donor 2 & Donor 1 \\
\hline Treated RBC & Chip 5 & Donor 1 & Donor 2 & Donor 1 & Donor 2 \\
\hline Treated RBC & Chip 6 & Donor 2 & Donor 1 & Donor 2 & Donor 1 \\
\hline
\end{tabular}




\section{APPENDIX B: Electronic Lab Notebook}

\section{7 location of hydrophobic coating on DropBot chip}

As shown in recorded images, there is a visible edge to the hydrophobic layer on the chip. A line showing the edge of the hydrophobic layer is seen about halfway between the reservoirs and the innermost contact pads, running parallel to the short edge of the chip. DropBot chip used was f7660e8e-1872-43f8-ab23-267de2c4759a (759a).

20180921 droplet actuation on DropBot chip with propylene glycol

Used propylene glycol on (new) DropBot frame with chip 759a. Achieved full droplet control (creation, motion, etc.) with $100 \mathrm{~V}$. Videos were captured using the AmScope MU8000.

Videos recorded in Google Drive.

20180927 attempted droplet "clotting test" over electrode

Previously discussed was a method to test sample clotting state by moving the droplet sample over a single electrode, de-actuating to recover a circular shape, then actuating two adjacent electrodes on either side to deform the droplet. The metric for clotting state would be the ratio of minor chord length to original droplet diameter.

An attempt to actuate the droplet as such was unsuccessful.

Used chip f7660e8e-1872-43f8-ab23-267de2c4759a (759a)

20181004 wetting angle of propylene glycol and blood on DropBot chip

Took images of DropBot provided propylene glycol to measure wetting angle. Four droplets of $3 \mathrm{ul}$ were placed on the uncoated section of the chip and four were placed on the coated section. Attempted to obtain blood using Ergo Lance high flow needle, but was unable to acquire enough blood to place eight droplets. One droplet of about $0.4 \mathrm{ul}$ was obtained and measured. Wetting angle was obtained using ImageJ dropsnake LBDSA plugin. Chip 759a was used. 
Videos recorded in Google Drive.

20181011 wetting angle of blood on DropBot chip

Took images of blood on DropBot chip 759a. Used BD Microtainer Contact-Activated Lancet. Dispensed four droplets of $\sim 0.6 \mathrm{ul}$ on hydrophobic surface of chip. Measured using DropSnake LDBSA method.

Videos recorded in Google Drive.

20181011 established protocol to create a four-electrode droplet segment with propylene glycol

Used trial-and-error methods to establish DropBot protocol.

20181015 attempted to verify protocol and test new chip

Attempted to verify protocol previously established. DropBot's onboard self-tests registered no shorts or channel errors. However, on chip 759a, reservoir electrode 9 failed to actuate. Other reservoirs saw actuation but electrodes leading to the main body of electrodes were unable to see actuation. Tested chip 186afba5-206d-46c5-b8a6-8c681c68cda8 (cda8). Reservoir electrode 9 saw actuation as normal, attempted to establish new protocol. Observed that body electrodes beneath the "horizontal" line bisecting the short dimension of the chip did not see actuation.

20181029 established droplet splitting protocol for four-electrode segment

Used propylene glycol on chip 759a. Established procedure starting from four-electrode segment to six-electrode split. Tested at two reservoir pairs, one segment saw a complete split and one did not. Split at $110 \mathrm{~V}$, which was very quick compared to $100 \mathrm{~V}$ or $95 \mathrm{~V}$.

20181029 attempted actuation with blood

Achieved actuation from reservoir at $100 \mathrm{~V}$. Unable to actuate past two electrodes from reservoir. Turned voltage up to $110 \mathrm{~V}$, and achieved actuation and droplet split per previous protocol. 
20181102 determined volume for four-electrode droplet segment

Used propylene glycol to experimentally determine volume for four-electrode droplet segment on chip 759a. The goal was to dispense fluid into the reservoir and use this fluid as the segment, rather than split a portion of fluid from a larger amount dispensed in the reservoir. Verified DropBot DB3 120 chip to have electrodes $2.2 \mathrm{~mm} \times 2.2 \mathrm{~mm}$ with vertical gap spacing (between plates) of $180 \mathrm{um}$. As such, the volume of the four-electrode rounded rectangle should be slightly under $3.48 \mathrm{~mm}^{3}$.

Attempted actuation from reservoir 85 and 34 at $110 \mathrm{~V}$ seeing difficulty (perhaps due to flaking hydrophobic coating at the edge of the top plate). The fluid visibly spread along the edge of the top plate, past the edges of the reservoirs. Achieved actuation with reservoir 93 at 120V, which was previously used for blood actuation, with $5 \mathrm{ul}$ dispensed. Once the droplet moved several electrodes from the reservoir, actuation voltage was reduced to $110 \mathrm{~V}$ and proceeded as normal. Visibly, the droplet was "caught" on the edge of the top plate, and a small amount of fluid remained and broke off from the main volume. The volume of the four-electrode segment was thus determined experimentally to be $5 \mathrm{ul}$, although it is likely necessary to dispense 5.1 or $5.2 \mathrm{ul}$ to account for fluid left in the reservoir.

20181108 attempted to assess clotting of diluted fresh frozen plasma (PRP) using thrombin

Collected vial of 50ul of thrombin and vial of PRP from CP at 12:40. Kept thrombin on ice and thawed PRP. CP suggested thrombin-PRP ratio of 1:50 by volume. Dispensed 500 ul of PBS, $25 \mathrm{ul}$ of PRP, and $0.5 \mathrm{ul}$ of thrombin, mixed by pipetting. Attempted to assess clotting with pipette tip. Perhaps due to the dilution or small tube, it was unclear if any clotting occurred. In a second tube, dispensed 50 ul of PRP and $\sim 2$ ul of thrombin. Mixed with pipette. Attempted to assess clotting with pipette tip. Some gelling was visible, but due to the small volume of fluid, changes over time were not observable.

Thrombin: https://www.fishersci.com/shop/products/thrombin-restriction-grade/696713

20181108 actuation of thrombin

Attempted actuation of thrombin on chip 759a. Set actuation voltage to $110 \mathrm{~V}$, dispensed $\sim 5 \mathrm{ul}$ of thrombin in reservoir 9 . Fluid was not pulled under top plate, so used pipette to remove fluid and dispensed in reservoir 70, which successfully pulled the fluid under the top plate. Attempted to actuate the thrombin across the chip towards reservoir 49. Successfully pulled a three-electrode segment of fluid upon increasing voltage to $130 \mathrm{~V}$. 
Thrombin: https://www.fishersci.com/shop/products/thrombin-restriction-grade/696713 $25 \mathrm{U} / \mathrm{ml}$.

Videos recorded in Google Drive.

20181113 off-chip clotting testing with plasma and thrombin

Acquired PRP and thrombin from CP at 11:30 am. Immediately placed both in freezer section of E115 refrigerator. Took PRP out at 1:10 pm to thaw. Diluted $100 \mathrm{ul}$ of PRP 1:10 with PBS in one tube and placed $100 \mathrm{ul}$ of PRP in another. Added $2 \mathrm{ul}$ of thrombin to each tube and mixed by pipetting several times. The pure PRP-thrombin visibly retained air bubbles upon mixing.

Attempted to test gel action by placing droplets on glass slides, then holding the slide at an angle to observe the droplet's motion on the slide. Droplets from diluted PRP and pure PRP were indistinguishable by sight. Both flowed down the glass slide freely, with a very small wetting angle.

Combined $200 \mathrm{ul}$ of PRP with $5 \mathrm{ul}$ of thrombin and mixed by pipetting. Mixture appeared to gel by sticking on the pipette tip. A droplet from this sample was placed on a glass slide, and the motion upon tilting was indistinguishable from either other sample, flowing freely down the slide and showing no evidence of gelling.

A possible cause for this is the hydrophilicity of PRP on the glass slide.

20181126 off-chip clotting testing with plasma and thrombin

Obtained platelet-rich plasma (PRP) frozen, $\mathrm{CaC} 2$ (diluted in what?), and thrombin from $\mathrm{CP}$ at 12:00 pm. In four-contained clot tray, mixed four samples. Each sample contained $100 \mathrm{ul}$ of PRP, $2 \mathrm{ul}$ of $\mathrm{CaC} 2$, and $2 \mathrm{ul}$ of thrombin. Samples had $0 \mathrm{ul}, 50 \mathrm{ul}, 100 \mathrm{ul}$, and $200 \mathrm{ul}$ of DI water to dilute the PRP. Samples were made by dispensing the DI water into each tray, then dispensing the PRP into each tray. $\mathrm{CaC} 2$, then thrombin was then dispensed into each tray. Mixing was done by pipette, taking the sample into the pipette and re-dispensing. The rapid clotting time presented a mixing challenge, as only one sample could be mixed at a time. As such, the $50 \mathrm{ul}$ and 100 ul-diluted samples were noticeably less homogenous than the $0 \mathrm{ul}$ and $200 \mathrm{ul}$ samples. The mixing time was also extended so as to not introduce air bubbles into the samples. 
After $\sim 1$ minute, gelling was apparent by sight in the undiluted sample. After $\sim 5$ minutes, gelling was apparent in all samples, including the sample diluted by $200 \mathrm{ul}$ of water.

After $\sim 15$ minutes, samples were prodded using a stirrer. Especially in the $100 \mathrm{ul}$ and 200 ul-diluted samples, it appeared that the clots formed were very non-homogeneous, forming a gel mass with surrounding liquid. After $\sim 20$ minutes, a visibly opaque white mass $\sim 6 \mathrm{~mm}$ in diameter was observed in the $50 \mathrm{ul}$ sample.

20181203 off-chip clotting testing with plasma and thrombin

PRP obtained from CP at 12:35. Used 100 ul of plasma in each sample. Dispensed water, then plasma and mixed by pipette. Dispensed $2 \mathrm{ul}$ of calcium, then $2 \mathrm{ul}$ of thrombin. Mixed by pipette. Using pipette tip, no clotting action was visible. To verify materials, dispensed $200 \mathrm{ul}$ of plasma, $5 \mathrm{ul}$ of calcium, and $5 \mathrm{ul}$ of thrombin and mixed by pipette. Clotting was observed. Waited 5 minutes and placed specimens on angled glass slide to observe wetting action. No differences between samples were observed.

\begin{tabular}{|l|l|l|}
\hline Specimen No. & Dilution & Water vol. \\
\hline 1 & $1: 5$ & $.5 \mathrm{ml}$ \\
\hline 2 & $1: 8$ & $.8 \mathrm{ml}$ \\
\hline 3 & $1: 12$ & $1.2 \mathrm{ml}$ \\
\hline 4 & $1: 15$ & $1.5 \mathrm{ml}$ \\
\hline
\end{tabular}

2019114 on chip specimen creation and mixing protocol

Chip c4c5. Mixed 5 ul FFP with 10 ul PBS, and in a separate container, 2 ul Ca02 and 2 ul thrombin. Did not add surfactant. Did not successfully actuate on chip as the FFP-PBS mixture would not suck under the top plate. Tested reservoir 9 vertical pair with provided propylene glycol sample fluid and achieved actuation from the lower reservoir to reservoir 9.

At this time, the thrombin and plasma were too old $(\sim 1 \mathrm{hr})$ to continue clotting so attempted to determine on-chip plasma actuation dilution. Created specimens at 1:4, 1:5, 1:8, and 1:10 dilution (all with $20 \mathrm{ul}$ of FFP). Added $\sim 0.5 \mathrm{ul}$ of surfactant, but amounts are imprecise due to surfactant viscosity. Achieved actuation with $100 \mathrm{~V}$ of 1:4 dilution, but later required $120 \mathrm{~V}$ to actuate and the specimen gradually slowed in actuatability 
(perhaps dried out?). Achieved actuation of 1:5 dilution with $120 \mathrm{~V}$, the specimen gradually slowed in actuatability, switched to $130 \mathrm{~V}$, the specimen was eventually not actuatable with $130 \mathrm{~V}$.

Video of FFP 1:5 actuation recorded in Google Drive.

Video of FFP 1:4 actuation recorded in Google Drive. Surfactant is Tetronic 90R.

20190117 on-chip FFP pull tests

Chip c4c5. Created four FFP specimen concentrations at 1:8 (20 ul FFP, $160 \mathrm{ul}$ PBS), 1:12 (20 ul FFP, 240 ul PBS), 1:16 (20 ul FFP, 320 ul PBS), 1:20 (20 ul FFP, 400 ul PBS). Attempted to add $0.1 \%$ by volume surfactant to each specimen, but the viscosity of the surfactant means the additions were very imprecise. Mixed using vortex mixer for 15 $\mathrm{s}$ at $3000 \mathrm{rpm}$. Unable to pull FFP. Began at $80 \mathrm{~V}$ and increased to $120 \mathrm{~V}$. Actuation became difficult as time went on and eventually specimens stopped moving. Became visually cloudy

20190130 on-chip Whole blood pull tests

Chip c4c5. Created blood specimen with 40 ul whole blood, 800 ul PBS, $\sim 1$ ul Tetronic 90R4. Mixed by pipette, completed $\sim 30$ pumps at 150 ul. Actuated on reservoir 26 at 80 V. Specimen was easily sucked under top plate and actuated, albeit slowly, at 80V. Specimen was $\sim 5$ electrodes so a pull test over an additional electrodes was conducted. Unable to pull specimen at $80 \mathrm{~V}$, so went up to $90 \mathrm{~V}$. Still unable to pull, so went to 100 V. Specimen pulled to characteristic "hourglass" shape, but visibly separated. Pull was much slower than that of PBS or previous whole blood at $120 \mathrm{~V}$. Previous whole blood specimen, also diluted 1:20, was mixed with vortexer. After returning blood to reservoir 26 , could not extract with pipette, cleaned by wicking.

Created blood specimen with 80 ul whole blood, 800 ul PBS $<\sim 1$ ul Tetronic 90R4. Mixed by pipette, completed, 30 pumps at 180 ul. Actuated on reservoir 93. Pulled whole specimen over four segments at $80 \mathrm{~V}$. Went to $90 \mathrm{~V}$ at 5:25. Pull tested at $100 \mathrm{~V}$. Went to $110 \mathrm{~V}$ at 9:05. Blood noticeably separated. At 19:10 went to $90 \mathrm{~V}$, at 19:41 went to $120 \mathrm{~V}, 20: 31$, went to $80 \mathrm{~V}$. Unable to return specimen to reservoir, so removed by wicking.

1:20 Videos recorded in Google Drive.

1:10 Videos recorded in Google Drive. 
20190201 on-chip Whole blood pull tests

Attempted to actuate whole blood undiluted. Used whole blood which was $\sim 54$ hours old. Combined $\sim 160$ ul whole blood with $\sim 1$ ul surfactant, mixed by pipette. During mixing blood and surfactant, a gel substance was noticeable, potentially made of the surfactant (very viscous) combined with the blood. Attempted several times on reservoirs 93, 26, and the both of the pair to the left. Voltages ranged from $80 \mathrm{~V}$ to $120 \mathrm{~V}$. Blood would easily slip under top plate, and would sometimes actuate to first electrode, but would stop at second electrode and eventually stopped responding to actuation. This may be because the surfactant gels when mixed with the blood, causing the blood to not reduce in surface tension.

Videos recorded in Google Drive.

20190206 on-chip Whole blood pull tests

Chip c4c5. Suspected short at electrode 82, 66, 21, reserv. 70, 34. Tested with propylene glycol and verified no actuation on $82,66,38$, reserv. 34 . Switched to chip cda8.

Suspected on cda8: $67,83,99,110,114$. With propylene glycol, verified no actuation on $67,83,99,110,114$. Inserted test chip and ran all onboard tests, which verified no shorts and no open circuits.

Inserted chip 4540. Restarted MicroDrop. Attempted actuation with sample fluid. Verified actuation on 49-70 (rightmost) reservoir pair. Restarted MicroDrop and inserted chip c4c5. Attempted actuation on reservoir 9 to electrode 10 and reservoir 26 to electrode 20 with no response. Reservoir 34 did not show response, but electrode 36 actuated the sample fluid. Electrode 37 showed no response.

Quit MicroDrop software. Inserted chip 4540. Attempted actuation with sample fluid. Achieved actuation.

20190212 on-chip Whole blood pull tests

Used chip 4540. Created whole blood specimens with $1 \mathrm{ml}$ PBS, 20 ul whole blood, $0.1 \%$ surfactant Tetronic 90R4. Attempted actuation

Inserted chip cda8. Attempted actuation from reservoir 110 at $100 \mathrm{~V}$. Verified actuation. Actuated whole blood from reservoir 110 at $100 \mathrm{~V}$. Saw rapid actuation into test zone, performed pull test successfully. 
Actuated whole blood from reservoir 93. Also saw rapid actuation, ran test. Had forgotten to start video, so quit test, started video, and performed test again. The specimen deformed noticeably more slowly than the first unrecorded test. Specimen saw slow actuation back to reservoir.

Attempted actuation from reservoir 85. Actuated specimen to position and performed pull test. Was noticeably slower than prior two pull tests. Unable to return specimen to reservoir. Had to wick specimen out of chip.

Attempted actuation from reservoir 70. Quickly actuated specimen to test zone. Initiated pull test, which was noticeably slower than the prior 3 tests. Unable to return specimen to reservoir, had to wick.

Again attempted actuation on reservoir 110. Successfully pulled specimen into zone at $105 \mathrm{~V}$. Attempted test, but specimen appeared immobile. Actuated back to reservoir at $120 \mathrm{~V}$.

Made sure to regularly mix specimens by pipette.

20190214 on-chip PG pull tests

Chip cda8.

PG_2R1_021419cda8.mpg pull at 00:17. Pulls on propylene glycol were inconsistent and challenging, suggesting biofouling and residue playing a significant role in actuation difficulty. Will wait for new chips and achieve data from propylene glycol.

20190219 on-chip PG pull tests for repeatability and reproducibility

Chip 4dda. Attempted pulls on propylene glycol at $100 \mathrm{~V}$. Dispensed $5.5 \mathrm{ul}$ of PG in all reservoirs. Could not purge air from pipette so inconsistent dispensed volume. Added $\sim 1$ ul to middle two specimens. Actuated all specimens into pull test position at $90 \mathrm{~V}$. Pulled specimens simultaneously at $100 \mathrm{~V}$. Returned to position at $90 \mathrm{~V}$ for $\sim 1 \mathrm{~min} 30 \mathrm{~s}$ and repeated pull three times. Residue (?) was visible on sides of specimens.

Returned specimens to original reservoirs. Manipulated droplets to clean off residue. Moved specimens to upper reservoirs, rather than lower. Some specimens actuated to test position quicker (as before), but made sure to turn off electrodes for resting specimens so as to avoid residue formation. Briefly increased voltage to $110 \mathrm{~V}$ in order to pull specimen 1 from reservoir. Held specimens in positions at $100 \mathrm{~V}$ for $\sim 30 \mathrm{~s}$ then pulled at $100 \mathrm{~V}$. Repeated pull three times as previous. All specimens (except position 2) necked only on left side, with the right edge remaining straight.

PGR1_4dda_021919.mp4 
Pull 1: 07:55

Neutral 1: 17:55

Pull 2: 19:30

Neutral 2: 26:40

Pull 3: 28:30

N3: $36: 22$

(VOID) PGR2_4dda_021919.mp4

Pull 1: 1:00

N1: $6: 40$

P2: 7:40

N2: $12: 48$

P3: $13: 48$

N3: $18: 48$

20190220 on-chip PG pull tests for repeatability and reproducibility

Used chip 4dda. Dispensed 5.75 ul of propylene glycol (PG) into all four "bottom-side" reservoirs. Actuated at $100 \mathrm{~V}$. Used $110 \mathrm{~V}$ briefly to pull specimen from reservoir in lane 4. Tried to "wipe down" residue from PG at sites using on-chip PG specimens. Brought specimens back to reservoirs and removed by pipette.

PGR3_4dda_022019.mp4

P1 - 2:10

P2 - 9:10

P3 - 15:30

Dispensed $5.75 \mathrm{ul}$ of fluid into each reservoir. Actuated into position at $110 \mathrm{~V}$. Pulled specimens at $110 \mathrm{~V}$. Made two mistakes: the specimen at lane 4 split and it was not reassembled between pulls 1 and 2, and two replicates were completed rather than three. Returned specimens to reservoir, wiping down residue and removing specimens by pipette. Dispensed 5.75 ul of new sample fluid. Completed three replicates, pulling at 110 V. Removed specimens as described above. Dispensed 5.75 ul of new sample fluid, completed an additional three replicates pulling at $110 \mathrm{~V}$ and removing specimens as described above.

PG110VR1_4dda_022019.mp4

P1 - 3:00

$\mathrm{P} 2-9: 50$

P3 - 16:31 
PG110VR2_4dda_022019.mp4

$\mathrm{P} 1-3: 52$

$\mathrm{P} 2-10: 00$

P3 - 16:00

20190305 on-chip thrombin actuation attempt

Used chip 4540. Obtained $\sim 20$ ul thrombin from E203. Added in $\sim 0.1 \%$ Tetronic 90R4 and mixed by vortex. Volume of added Tetronic 90R4 is approximate due to high viscosity. Attempted actuation on leftmost bottom reservoir. Dispensed $\sim 5 \mathrm{ul}$ and attempted actuation. Thrombin specimen got "stuck" so added $\sim 4$ ul of thrombin specimen. Varied voltage from 100V - 120V, achieved some actuation. "Unstuck" thrombin from top plate edge at reservoir, but specimen remained stuck within first electrode in grid. Pulled specimen successfully back to reservoir. Chip 4540 has seen fouling so this may be the cause.

Actuation video record THR_030529.mp4 in Google Drive.

20190308 on-chip thrombin actuation attempt

Initiator was 50 ul calcium, 8 ul thrombin, $\sim 0.1 \%$ Tetronic $90 \mathrm{R} 4$ surfactant. Blood was $1.5 \mathrm{ml}$ whole blood $+0.1 \%$ surfactant.

Chip 4540 100-100V, 2000-10000 Hz. Thrombin quickly clouded over visually, "froze" at initial electrodes after a couple minutes.

Chip 4dda, dispensed $\sim 5$ ul of thrombin+calcium initiator mixture. Actuated initiator at $5000 \mathrm{~Hz} 80 \mathrm{~V}$ from reservoir (top row, second from left). Moved initiator into lane, centered between reservoirs.

Went up to $100 \mathrm{~V}$ at 6:27. Dispensed $\sim 6$ ul blood into reservoir 93(?). Moved initiator adjacent to blood. Initiator and blood droplet specimens were initially adjacent but not merged. After a minute or so, the droplets merged. Turned off all electrodes at 7:58. Waited $\sim 20$ min for clot to develop.

Attempted to actuate excess initiator away from blood but unsuccessful. Initiator appears "stuck" on a particle or "gel line." This could be surfactant? Attempted to remove blood by pipette using PBS to break surface tension. Blood appeared fully clotted, or perhaps dried. Attempted to wick blood out. The blood at the top-bottom plate reservoir entrance acted as a solid barrier for wicking Kimwipes. Video is CLOT_030819.mp4 but has compression issues? 
20190327 on-chip PBS pulls (with fluorescein)

Chip 82949ADC-28A6-11E9-BDCE-9CB6D0E73EB4. Placed such that text "SCI-BOTS 90-pin array v3" was not placed against back of DropBot. Added $500 \mathrm{ul}$ of PBS, added $0.0262 \mathrm{~g}$ of Tetronic 90R4 on scale. Added an additional $1000 \mathrm{ul}$ of PBS and $75 \mathrm{ul}$ of fluorescein. Conducted tests with homemade "darkbox" over camera. Dispensed 5.5 ul of specimen into each "bottom" reservoir and actuated all specimens into position by hand. Pulled specimens simultaneously at at $100 \mathrm{~V}$ using protocol "Pull test $100 \mathrm{~V}$ ".

Video recorded in Google Drive.

20190328 on-chip PBS pulls (with fluorescein)

Chip 82949ACF-25A5-11E9-B21B-9CB6D0E37EB4. Dispensed PBS specimen created on 03-27-19. Dispensed 5.5 ul of specimen on each bottom reservoir on chip. Put "dark box" over DropBot. Actuated all specimens into test sites. Pulled all specimens at $100 \mathrm{~V}$. Tests were noticeably slower than the previous tests, with only two specimens fully splitting. Rightmost specimen was noticeably larger in area than other specimens.

Video recorded in Google Drive.

20190507 on-chip RBC pulls to verify hematocrit \%

Chip 82949ACF-28A6-11E9-B218-9CB6D0E37EB4

For $\mathrm{H}=2.5 \%, 1.462 \mathrm{ml}$ PBS, 38 ul blood.

For $\mathrm{H}=5 \%, 1.425 \mathrm{ml}$ PBS, 75 ul blood.

$\mathrm{H}=10 \%, 1.350 \mathrm{ml}$ PBS, 150 ul blood.

All specimens were mixed by pipette at between 10 and 20 times. $\mathrm{H}=2.5 \%$ was tested first, actuated at $100 \mathrm{~V}$ and saw a strong response and successful pull test. $\mathrm{H}=5 \%$ was tested next, actuated at $100 \mathrm{~V}$ and saw a strong response and successful pull test. $\mathrm{H}=10 \%$ was tested last, actuated $100 \mathrm{~V}$, was slightly slower to leave the reservoir, but saw a successful pull test.

Video recorded in Google Drive.

20190510 on-chip RBC pulls 
One donor used. Created samples at $10 \%$ hematocrit. Centrifuged whole blood at $250 \mathrm{rcf}$, acc 7, break 0, time $20 \mathrm{~min}$, (collected $\sim 1$ hour before), pulled and disposed plasma and WBC layer with pipette. Added $500 \mathrm{ul} \mathrm{RBCs}$ and $4500 \mathrm{ul}$ PBS to a tube and centrifuged at same settings to wash. Removed and disposed of PBS by pipette.

Created samples at 10\% hematocrit by dispensing $900 \mathrm{ul} \mathrm{PBS}$ and $0.2 \%$ (of $1 \mathrm{ml}$ ) surfactant into a microcentrifuge tube. Mixed using a vortex mixer at $3000 \mathrm{rpm}$ for $15 \mathrm{~s}$. Dispensed 100 ul of washed RBC

Inserted chip 1 (EC484FB9-03C5-11E9-816F-9CB6D0E37EB4) in "up" orientation ("SCI-BOTS" patterned text on glass is towards the back of the DropBot). Chip 2 is EC484FC2-03C5-11E9-835D-9CB6D0E37EB4.

C1L1 Pull at 0:58

C1L3 Pull at 0:50

\section{4 on-chip RBC pulls}

Chip 4, electrode 18 not addressable

Chip 3 - EC4B28C8-03C5-11E9-93B8-9CB6O0E37EB4

\section{On-chip pulls}

Weighed $0.035 \mathrm{~g}$ of surfactant, added $15.2 \mathrm{ml}$ of PBS to achieve $0.2 \%$ by volume (for $90 \%$ PBS, $10 \%$ RBCs). Mixed by vortex.

C3L2 - Electrodes 26, 21, 98 not actuating.

\begin{tabular}{|l|l|l|l|l|}
\hline Chip 7 & Untreated & Untreated & Treated & Treated \\
\hline Chip 8 & Treated & Treated & Untreated & Untreated \\
\hline
\end{tabular}

